\title{
Placental methylome reveals a 22q13.33 brain regulatory gene locus associated with autism
}

Yihui Zhu' ${ }^{1,2,3,4}$, J. Antonio Gomez ${ }^{1,2,3,4}$, Benjamin I. Laufer ${ }^{1,2,3,4}$, Charles E. Mordaunt 1,2,3,4, Julia S. Mouat 1,2,3,4, Daniela C. Soto 3,4,5, Megan Y. Dennis ${ }^{3,4,5}$, Kelly S. Benke ${ }^{6}$, Kelly M. Bakulski ${ }^{7}$, John Dou', Ria Marathe ${ }^{1,2,3,4}$, Julia M. Jianu 1,2,3,4, Logan A. Williams 1,2,3,4, Orangel J. Gutierrez Fugón ${ }^{1,2,3,4}$, Cheryl K. Walker ${ }^{2,4,8}$, Sally Ozonoff, ${ }^{4,9}$ Jason Daniels ${ }^{10}$, Luke P. Grosvenor ${ }^{10}$, Heather E. Volk ${ }^{10,11}$, Jason I. Feinberg ${ }^{11}$, M. Daniele Fallin ${ }^{11}$, Irva Hertz-Picciotto ${ }^{2,4,6}$, Rebecca J. Schmidt ${ }^{2,4,6}$, Dag H. Yasui ${ }^{1,2,3,4}$ and Janine M. LaSalle ${ }^{1,2,3,4^{*}}$ (i)

* Correspondence: jmlasalle@ ucdavis.edu

'Department of Medical Microbiology and Immunology, University of California, Davis, CA USA

${ }^{2}$ Perinatal Origins of Disparities Center, University of California, Davis, CA, USA

Full list of author information is available at the end of the article

\begin{abstract}
Background: Autism spectrum disorder (ASD) involves complex genetics interacting with the perinatal environment, complicating the discovery of common genetic risk. The epigenetic layer of DNA methylation shows dynamic developmental changes and molecular memory of in utero experiences, particularly in placenta, a fetal tissue discarded at birth. However, current array-based methods to identify novel ASD risk genes lack coverage of the most structurally and epigenetically variable regions of the human genome.
\end{abstract}

Results: We use whole genome bisulfite sequencing in placenta samples from prospective ASD studies to discover a previously uncharacterized ASD risk gene, LOC105373085, renamed NHIP. Out of 134 differentially methylated regions associated with ASD in placental samples, a cluster at 22q13.33 corresponds to a 118-kb hypomethylated block that replicates in two additional cohorts. Within this locus, NHIP is functionally characterized as a nuclear peptide-encoding transcript with high expression in brain, and increased expression following neuronal differentiation or hypoxia, but decreased expression in ASD placenta and brain. NHIP overexpression increases cellular proliferation and alters expression of genes regulating synapses and neurogenesis, overlapping significantly with known ASD risk genes and NHIPassociated genes in ASD brain. A common structural variant disrupting the proximity of NHIP to a fetal brain enhancer is associated with NHIP expression and methylation levels and ASD risk, demonstrating a common genetic influence.

Conclusions: Together, these results identify and initially characterize a novel environmentally responsive ASD risk gene relevant to brain development in a hitherto under-characterized region of the human genome.

Keywords: Autism spectrum disorder, Epigenomics, Human genetics, Structural variants, DNA methylation, Prospective study, Placenta, Hypoxia, Neurodevelopment, Postmortem brain

(c) The Author(s). 2022 Open Access This article is licensed under a Creative Commons Attribution 4.0 International License, which permits use, sharing, adaptation, distribution and reproduction in any medium or format, as long as you give appropriate credit to the original author(s) and the source, provide a link to the Creative Commons licence, and indicate if changes were made. The images or other third party material in this article are included in the article's Creative Commons licence, unless indicated otherwise in a credit line to the material. If material is not included in the article's Creative Commons licence and your intended use is not permitted by statutory regulation or exceeds the permitted use, you will need to obtain permission directly from the copyright holder. To view a copy of this licence, visit http://creativecommons.org/licenses/by/4.0/. The Creative Commons Public Domain Dedication waiver (http://creativecommons.org/publicdomain/zero/1.0/) applies to the data made available in this article, unless otherwise stated in a credit line to the data. 


\section{Background}

Autism spectrum disorders (ASD) are growing in prevalence, with 1 in 54 children diagnosed in the USA [1]. Diagnosis of ASD is based on a child's behavioral difficulties in social communication and interactions, language deficits, restricted interests and repetitive behaviors, and sensory sensitivities. The etiology of ASD is complex and heterogeneous, and it is likely to involve multiple genetic and environmental factors, as well as poorly understood gene-environment interactions [2-4]. Twin and sibling studies have shown a strong heritability of ASD risk within families, and most genetic risk for ASD is expected to come from common variants [5]. Exome sequencing of ASD trios has identified genes with rare mutations in ASD children, which are enriched for neuronal, embryonic development, and chromatin regulation functions, but no single gene explains more than $1 \%$ of disease risk [6, 7]. A large genome-wide association study (GWAS) identified individual genetic variants that contribute to an individual's ASD risk and showed that the weighed sum of the risk alleles and their effect sizes can be combined to create a polygenic risk score (PRS) for ASD [8]. The variance explained for ASD using this approach was $2.45 \%$, which was further improved to $3.77 \%$ by including in the prediction model additional PRS for traits co-heritable with ASD, including schizophrenia, depression, and educational attainment [8-10]. Common polygenic risk may also interplay with early environmental and perinatal factors in other neurodevelopmental disorders. For example, a PRS derived from a set of 108 previously identified genome-wide significant variants for schizophrenia [11] was shown to be significant only in the presence of early-life maternal complications (ELCs), and the subset of variants interacting with ELCs also corresponded with patterns of placental gene expression, consistent with the importance of placental gene regulation as a window into neurodevelopment [12]. However, most ASD genetic or environmental studies have not included placental molecular measures, despite the potential convergence between placental biology and genetic risk for ASD. Term placenta is an accessible tissue normally discarded at birth; however, the convergence between placental biology and genetic risk for ASD is relatively unexplored.

Placenta maintains a distinct landscape of DNA methylation characterized by partially methylated domains (PMDs), which is more similar to oocytes and preimplantation embryos, where methylation over gene bodies is positively correlated with expression, compared with the high overall methylated pattern of fetal or adult tissues [13-15]. In this way, placental methylation patterns are also similar to most epithelial-derived tumors, where PMDs are also found [16]. Both placenta and tumors show a strong responsiveness to hypoxia that promotes cell proliferation and angiogenesis, which are both required for invasion of the placental trophoblasts into the uterine decidua during placentation [17]. In vivo, hypoxia and reactive oxygen species promote neurogenesis in embryos, newborns, and adults, and also play a role in neuronal differentiation in vitro [18]. Furthermore, exposure to most environmental pollutants produces excessive oxidative stress that can impact both placentation and neurodevelopment [19].

Because of its multiple roles in support of fetal development during intrauterine life, the placenta is a promising tissue for identifying DNA methylation alterations at genes relevant to fetal brain and gene-environment interactions in ASD [20-23]. Most epigenome-wide association studies (EWAS) for ASD have used array-based methods to assess DNA methylation which lack coverage over the most epigenetically and 
genetically polymorphic regions of the human genome, such as correlated regions of systemic interindividual variation (CoRSIVs) and structural variants (SVs) [24]. CoRSIVs, which are regions of the genome with similar DNA methylation patterns across tissue types, are sensitive to periconceptional environment, observed across diverse tissues, associated with human disease genes, and are enriched for transposable elements and subtelomeric locations [24, 25]. SVs arising from transposable elements have been associated with many human phenotypes, especially immune responses and neuropsychiatric disorders, such as schizophrenia [26-28]. SVs exhibit a nonrandom distribution in hotspots within relatively gene-poor regions in primate genomes, but are enriched for gene functions in oxygen transport, sensory perception, synapse assembly, and antigen-binding $[29,30]$. Recent studies suggested that a large SV burden was associated with lower cognitive ability [31-33] and ASD [34], but most GWAS and EWAS studies ignore SVs and CoRSIVs in the genome. Therefore, the combination of utilizing the unique placental DNA methylation landscape reflective of in utero gene expression with sequencing-based epigenome-wide investigations inclusive of understudied genomic regions is warranted.

Here, we investigated the association of ASD risk with placental DNA methylation in two high-risk familial ASD cohorts through whole genome bisulfite sequencing (WGBS) analysis of 204 individuals. We identified a block of differential methylation in ASD at 22q13.33, a region previously described as a CoRSIV and SV hotspot but not previously associated with ASD. A novel gene LOC105373085 (renamed as NHIP for neuronal hypoxia inducible, placenta associated) within 22q13.33 was demonstrated to be expressed in brain, responsive to oxidative stress, and to influence expression of other known ASD-risk genes. A common SV insertion within 22q13.33 was associated with increased ASD risk, reduced expression of NHIP, and reduced methylation, but first month prenatal vitamin use counteracted this effect. Together, these results demonstrate a novel ASD risk gene regulatory locus at the interface of common genetics and perinatal environmental resilience.

\section{Results}

Differential methylation analysis using WGBS identifies a hypomethylated block at 22q13.33 in ASD placenta

To identify novel regions of epigenetic alterations in placenta discriminating later ASD diagnosis, we performed WGBS analysis of genome-wide DNA methylation on 204 subjects from two prospective high-risk ASD cohorts (MARBLES and EARLI) with a diagnosis outcome at 36 months (Fig. 1a). Subject clinical and demographic information as well as cell type proportions and global methylation measurements are provided in Additional File 1: Table S1, while data on individual sample sequencing information is in Additional File 2: Table S2. No demographic, cell type, or technical variables were significantly associated with ASD outcome, but scores related to ASD severity and cognition were associated, as expected (FDR-corrected and raw $p$ values from Fisher's exact for categorical or one-way ANOVA for continuous variables is in Additional File 1: Table S1). Global methylation levels over 20-kb windows were also not different by diagnostic group (Additional File 3: Fig. S1). Since sequencing platform differences (Illumina HiSeq 4000, HiSeq X, NovaSeq) impacted global methylation levels 


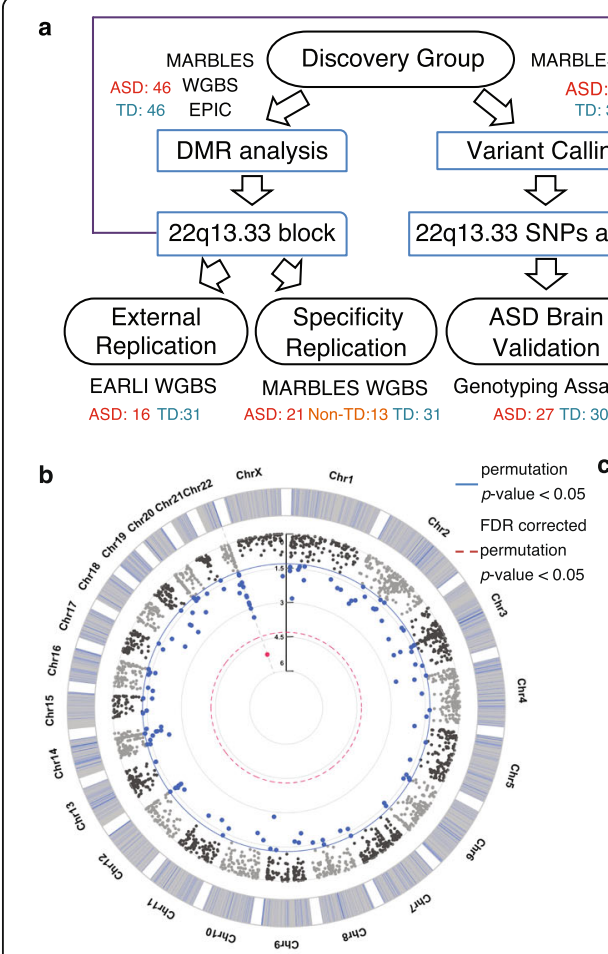

Histone Markers LES WGS TD: 37

\section{7}

Exp

Novel Transcript (NHIP)

ए qPCR

Expression in Tissues and Cells

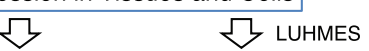

SV

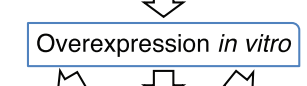

Response to Hypoxia

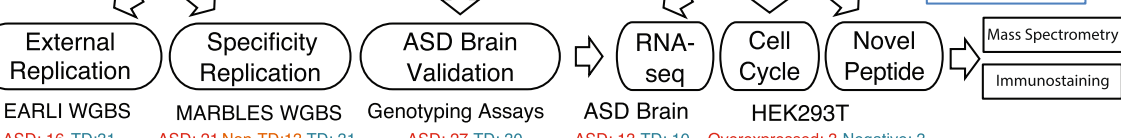

C ChromHMM predicted State

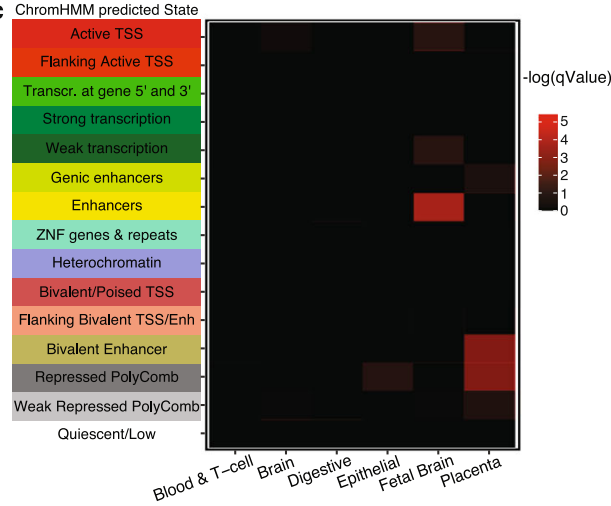

d

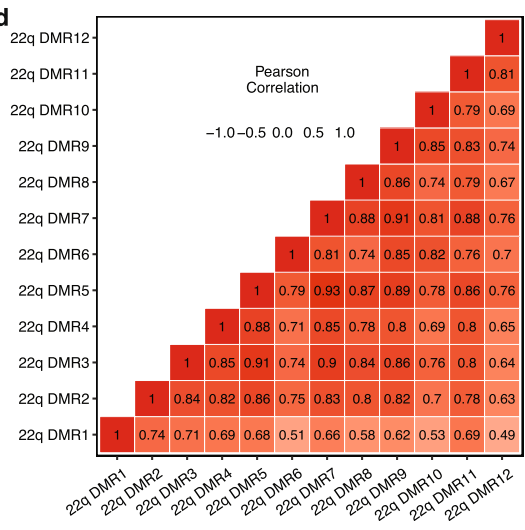

e Discovery Group External Replication Specificity Replication

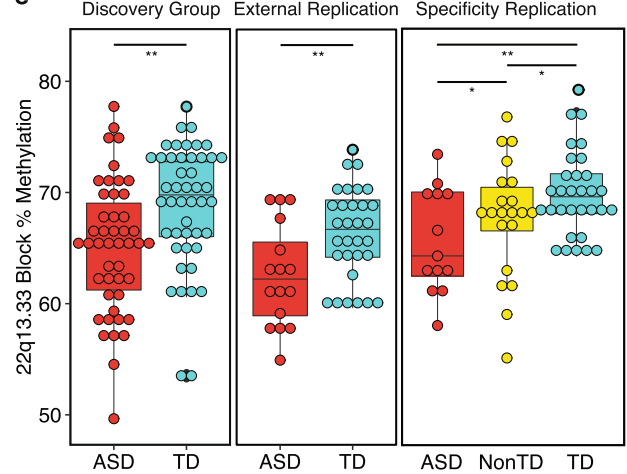

Fig. 1 ASD-associated DMRs are enriched at fetal brains enhancers and a co-methylated block at 22q13.33 replicates across studies and platforms. A Schematic of the experimental design for discovery of ASD DMRs, replication of the co-methylated 22q13.33 locus, genetic associations, and functional follow-up of a novel gene (NHIP). B Circular Manhattan plot of the epigenome-wide association of DNA methylation in placenta with ASD diagnosis at 36 months. Results are represented as DMR association test results $\left(-\log _{10}(p)\right)$. Significant thresholds are blue for permutation $p$ value $<0.05$, red for FDR-adjusted permutation $p$ value $<$ 0.05 , and gray for nonsignificant. C 134 ASD DMRs (permutation $p$ value $<0.05$ ) tested for enrichment within chromatin states defined by Epigenome Roadmap ChromHMM [35]. Each row represents a different ChromHMM state and each column a single tissue type, with the heatmap plotting the $-\log _{10}(q$-value) significance of ASD DMR enrichment. D Correlation matrix of methylation levels using the Pearson correlation coefficient for the 12 DMRs located in the 22q13.33 hypomethylated block. E Smoothed methylation values were averaged over the 22q13.33 hypomethylated block (y-axis) and compared across diagnosis groups ( $x$-axis). In the discovery group, ASD samples had significantly lower methylation than TD samples (MARBLES, HiSeq X, ASD $n=46$, TD $n=46$ ) ( $p$ value $=0.002$ ). The same result and direction were observed in the external replication group (EARLI, HiSeq 2500, ASD $n=16, \operatorname{TD} n=31$ ) ( $p$ value $=0.009$ ). For the specificity replication group (MARBLES, NovaSeq, ASD $n=21$, Non-TD $n=13, T D n=31$ ), ASD methylation levels were also significantly lower than both TD $(p$ value $=0.005)$ and Non-TD ( $p$ value $=$ 0.049), while Non-TD was significantly lower than TD samples ( $p$ value $=0.050$ ) by Mann-Whitney-Wilcoxon. Box plot center lines, box limits, and whiskers represented median, interquartile range, and minimum and maximum values, respectively 
(Additional File 3: Fig. S2), we separated the samples into "discovery," "external replication," and "specificity replication" groups for initial analyses of differentially methylated regions.

Differentially methylated regions (DMRs) distinguishing ASD from typical development (TD) placental samples were identified with a permutation-based statistical approach, adjusted for sex and placental cell types, to identify broad epigenomic signatures of multiple gene regulatory regions at a genome-wide level in the discovery group (Additional File 2: Table S2). In total, 134 DMRs (permutation $p$ value $<0.05$ ) representing an average size of 1027 bp with $5-10 \%$ smoothed methylation differences, including 77 hyper- and 57 hypomethylated in ASD compared to TD, mapped to 183 genes (Fig. 1b, DMR characteristics and gene names are in Additional File 4: Table S3). A cluster of 12 ASD DMRs mapped to 22q13.33, all hypomethylated in ASD (5-7\% difference from TD), including one that also passed genome-wide significance (FDR-adjusted $p$ value $<0.05$ ). Methylation levels within the 134 ASD DMRs were specifically associated with autism severity and cognitive scores, but not other demographic and technical variables after adjustment in a linear model (Additional File 3: Fig. S3). Further evidence that DMRs identified in placenta reflect epigenetic differences relevant to brain and development came from the significant enrichment of ASD DMRs in fetal brain enhancers, as well as bivalent enhancer and repressed polycomb regions of placenta compared to background regions using ChromHMM-defined chromatin states from the Roadmap Epigenomics Project [35] (Fig. 1c, detailed information on each tissue type and chromatin state is in Additional File 5: Table S4). Demonstrating their functional relevance, hyper-methylated ASD DMRs were enriched within 0-5-kb and 5-50-kb windows downstream of transcription start sites (TSS), at CpG islands and shores for both hyper- and hypomethylated DMRs (Additional File 3: Fig. S4-S5), and at known transcription factor binding sites (names, motifs, and enrichment statistics are in Additional File 6: Table S5). Genes mapping to placental ASD DMRs significantly overlapped with ASD risk genes from the Simons Foundation Autism Research Initiative (SFARI) dataset compared to all expressed genes (Fisher's exact test, $p$ value $=$ 0.006, odds ratio $(\mathrm{OR})=2.321$ ) [36] (Venn diagram in Additional File 3: Fig. S6, overlapping gene names and DMR characteristics are in Additional File 7: Table S6). The overrepresentation of 12 DMRs at 22q13.33 hypomethylated in ASD drove the additional enrichment at $>500 \mathrm{~kb}$ of TSS as well as gene ontology (GO) enrichment for functions in histone acetyltransferase (HATs) and chromatin modification, due to the assignment of the 22q13.33 hypomethylated DMRs to the nearest downstream gene $B R D 1$, a histone acetyltransferase (top Biological Process and Cellular Compartment GO terms are graphed in Additional File 3: Fig. S7, GO terms and enrichment statistics are in Additional File 8: Table S7). Based on the genome-wide significance and observation of multiple DMRs mapping to the same genomic region, we decided to focus subsequent analyses on further understanding the impact of the 22q13.33 hypomethylated locus on ASD risk.

The 22q13.33 DMRs hypomethylated in ASD were highly positively correlated with each other and formed a 118-kb hypomethylation cluster that was also detected as a hypomethylated block (chr22: 49044669 - 49162642, hg38) (Fig. 1d, plot of methylation values of individual samples is in Additional File 3: Fig. S8). This chromosomal locus was also previously described as a CoRSIV, a region of correlated methylation 
differences between individuals [24]. We therefore examined smoothed methylation levels over the $118 \mathrm{~kb} 22 \mathrm{q} 13.33$ block for replication in a different ASD enriched risk cohort (EARLI, external replication group). Similar to the discovery group (MannWhitney-Wilcoxon, $p$ value $=0.002$, ASD $n=46$, TD $n=46$, effect size $=-0.645$ ), 22q13.33 block methylation levels were significantly lower in ASD compared to TD (Mann-Whitney-Wilcoxon, $p$ value $=0.009$, ASD $n=16$, TD $n=31$, effect size $=$ -0.867) (Fig. 1e, comparisons of individual DMRs within the 22q13.33 block are in Additional File 9: Table S8). Furthermore, an independent "specificity replication group" of MARBLES subjects that included a "Non-TD" diagnostic group sequenced on a different platform also showed significantly lower 22q13.33 DNA methylation levels in ASD compared to either TD or the additional diagnostic Non-TD samples, defined as atypical cognitive scores but not ASD (Mann-Whitney-Wilcoxon, ASD vs TD $p$ value $=0.005$, effect size $=-0.991$, ASD vs Non-TD $p$ value $=0.049$, effect size $=$ -0.282 , Non-TD vs TD $p$ value $=0.050$, ASD $n=21$, Non-TD $n=13$, TD $n=31$, effect size $=-0.452)$ (Fig. 1e, Additional File 9: Table S8). Smoothed methylation levels over the 118-kb 22q13.33 block remained significantly associated with ASD after adjustment for 18 potential covariates in a linear model in the discovery group (Additional File 3: Fig. S3c) and after adjustment for two nominal covariates in all three groups (Additional File 3: Fig. S9 and Additional File 10: Table S9). These results demonstrate that hypomethylation over the $118 \mathrm{~kb} 22 \mathrm{q} 13.33$ co-methylated block is a reproducible finding across different cohorts and platforms, specifically distinguishing placental samples of newborns later diagnosed with ASD.

NHIP is a primate-specific gene dynamically expressed during neuronal differentiation that exhibits reduced expression in ASD

The 22q13.33 co-methylated block was within an apparent gene desert, located more than $500 \mathrm{~kb}$ away from the closest annotated protein coding genes: FAM19A5 (TAFA5) and BRD1. Epigenetic evidence for promoter and enhancer activity within 22q13.33 was obtained from placenta, ovary, and brain ENCODE datasets (Additional File 3: Fig. S10) [37]. Within 22q13.33, an active promoter peak identified by H3K4me3 histone markers was observed in a subset of ovary, placenta, and brain samples, suggesting variable promoter marks between individuals (Additional File 3: Fig. S11). This H3K4me3 peak overlapped a CpG island and the TSS of the uncharacterized transcript, LOC105373085 (also named AK057312) identified from a human testis cDNA library (Additional File 3: Fig. S11) [38]. We renamed LOC105373085 as NHIP, for neuronal hypoxia inducible, placenta associated. NHIP is also variably expressed among brain regions from the Genotype-Tissue Expression (GTEx) database (Additional File 3: Fig. S12) [39]. The full-length NHIP sequence is syntenic in all primates, but not in other vertebrates including mouse (Additional File 3: Fig. S13, conservation scores for individual species are in Additional File 11: Table S10). When quantified by RT-PCR in human tissues, NHIP was expressed in placenta, testis, and adult and fetal brain, with relatively lower expression in placenta (Fig. 2a). ASD placental samples showed significantly lower NHIP transcript levels than TD samples, in the same direction as methylation changes in the 22q13.33 block (Mann-Whitney-Wilcoxon, $p$ value $=0.009$, ASD $n$ $=17, \mathrm{TD} n=11$, effect size $=-1.12$, Fig. $2 \mathrm{~b})$. In placenta, because of the epigenetic 

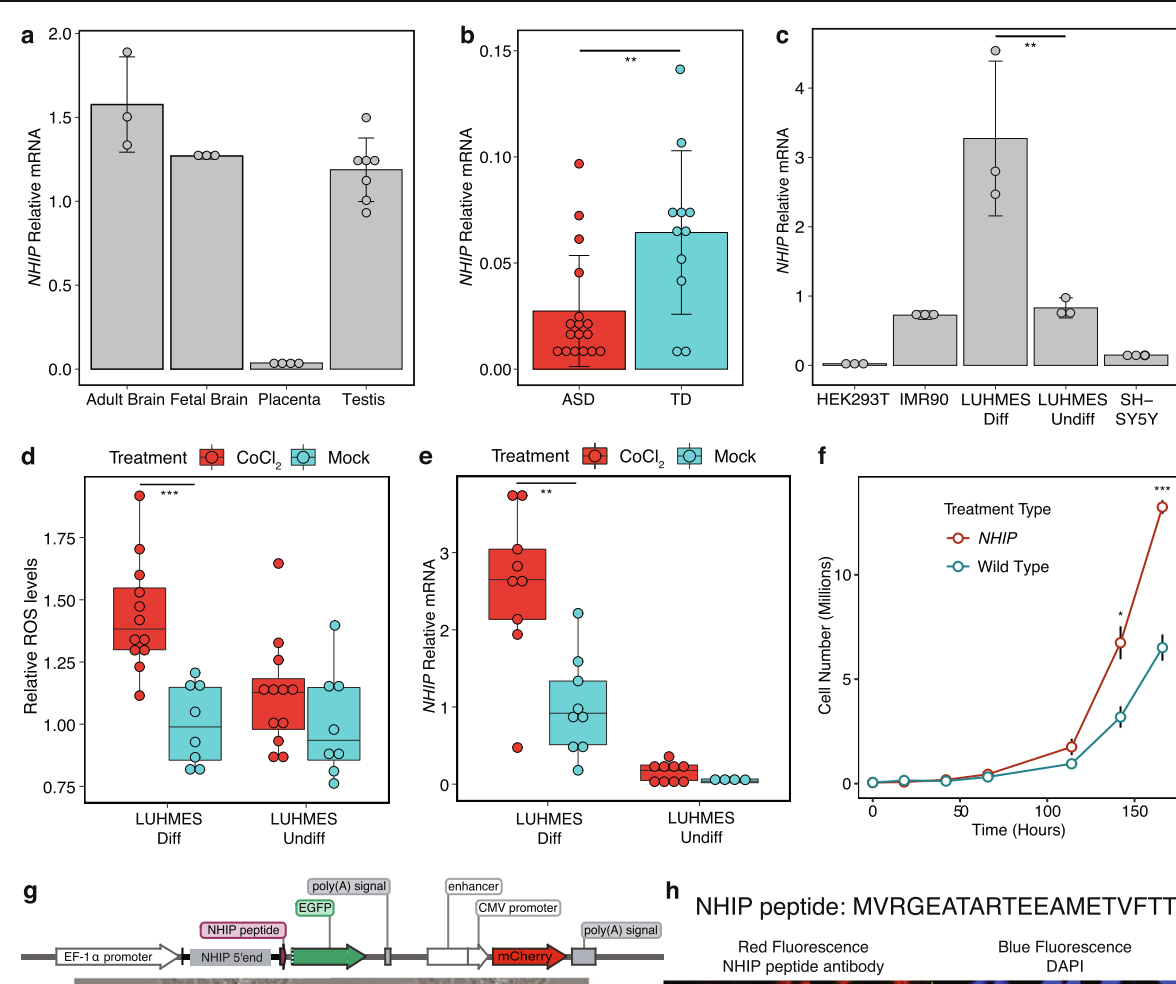

h NHIP peptide: MVRGEATARTEEAMETVFTT
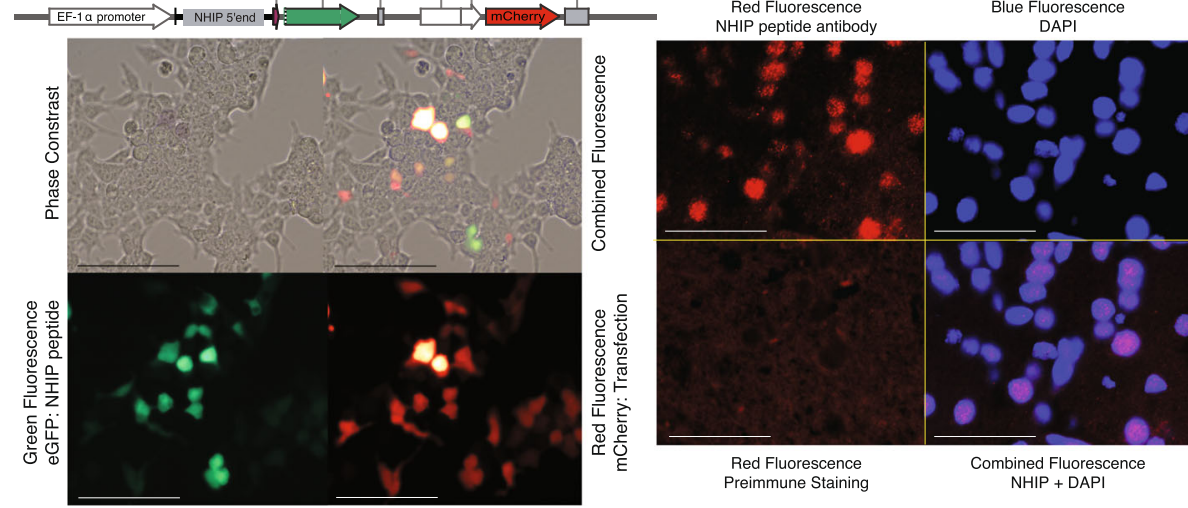

Fig. 2 Functional characterization of NHIP transcript levels in response to neuronal hypoxia. In a-e RT-qPCR assays, NHIP levels were normalized to GAPDH with at least three independent experiments per condition. A NHIP levels in human tissues, including adult brain, fetal brain, placenta, and testis. B NHIP levels in placenta samples from the discovery group (ASD $n=17, \mathrm{TD} n=11$ ). ASD samples show significantly lower NHIP levels than TD samples (Mann-Whitney-Wilcoxon, $p$ value $=0.009$ ). C NHIP levels in human cell lines, HEK293T, IMR90, LUHMES, and SHSY5Y. In LUHMES cells, NHIP levels were significantly higher in differentiated neurons compared to undifferentiated neurons (Mann-Whitney-Wilcoxon, $p$ value $=0.034$ ). $\mathbf{D}$ Differentiated LUHMES cells are more sensitive to hypoxia than undifferentiated LUHMES cells. Formation of reactive oxygen species (ROS) was measured in differentiated and undifferentiated LUHMES cells treated with $100 \mathrm{nM} \mathrm{CoCl}_{2}$, a hypoxia mimetic, or vehicle (mock) (MannWhitney-Wilcoxon, $p$ value $=0.0001$ ). E NHIP levels increase in response to hypoxia, specifically in differentiated neurons. Differentiated or undifferentiated LUHMES cells were treated with $100 \mathrm{nM} \mathrm{CoCl}_{2}$. In differentiated LUHMES cells, $\mathrm{CoCl}_{2}$ treatment significantly increased NHIP levels (Mann-Whitney-Wilcoxon, $p$ value $=0.009$ ). $\mathbf{F}$ NHIP overexpression in HEK293T cells resulted in a faster doubling time than vector control cells, indicating increased cell proliferation. (Hour 142, Mann-Whitney-Wilcoxon, $p$ value $=0.045$, NHIP overexpression cells $n=3$, control cells $n=3$, effect size $=3.10$; Hour 166, Mann-Whitney-Wilcoxon, $p$ value $=0.0009$, NHIP overexpression cells $n=3$, control cells $n=3$, effect size $=7.80$ ). $\mathbf{G}$ Vector design of NHIP peptide-eGFP (dotted line represents excised ATG of EGFP) and combined phase and fluorescent microscopy. Green, eGFP linked to NHIP peptide; red, mCherry, transfection positive control. Scale bars, 100 Mm. H Immunofluorescent staining of human frontal cortex, showing nuclear localization with anti-NHIP, but not pre-immune control. Blue, DAPI nuclear counterstain; red, anti-NHIP staining. Scale bars, $100 \mu \mathrm{m}$. Data are mean \pm SEM 
feature of PMDs, gene body methylation is positively associated with and predicts active gene expression across mammals $[13,15]$. Since the 22q13.33 co-methylated block mapped to a previously defined PMD in placenta [15] (map of region is in Additional File 3: Fig. S14), these results suggest that hypomethylation of the 22q13.33 block in ASD is reflective of lower past or current expression of NHIP expression in utero for ASD compared to TD.

To understand the function of this uncharacterized gene, we assayed and detected levels of NHIP expression in four human cell lines selected for their early-life origins (HEK293T, IMR90, LUHMES, SH-SY5Y). All cell lines are derived from human females; HEK293T cells are of embryonic kidney origin, IMR90 are fetal fibroblasts, Lund human mesencephalic (LUHMES) are derived from embryonic human mesencephalon, and SH-SY5Y cells are from a neuroblastoma. A significant increase in NHIP transcript levels was observed following neuronal differentiation in LUHMES cells (Mann-Whitney-Wilcoxon, $p$ value $=0.034$, differentiated LUHMES $n=3$, TD undifferentiated LUHMES $n=3$, effect size $=3.07$, Fig. $2 \mathrm{c}$ ). Since both neuronal differentiation and placental trophoblast differentiation respond to hypoxic conditions and oxidative stress in response to environmental pollutants [40, 41], we tested the responsiveness of NHIP to hypoxia. Differentiated LUHMES neurons were more sensitive to treatment with a hypoxia mimetic $\left(\mathrm{CoCl}_{2}\right)$ than undifferentiated cells, with a significant decrease in cell viability and an increase in reactive oxygen species (ROS) levels (Mann-WhitneyWilcoxon, $p$ value $=0.0001$, differentiated LUHMES treated with $100 \mathrm{nM} \mathrm{CoCl}_{2} n=$ 12, differentiated LUHMES treated with vehicle $n=8$, effect size $=2.26$, Fig. $2 \mathrm{~d}, \mathrm{H}_{2} \mathrm{O}_{2}$ results and statistics are in Additional File 3: Fig. S15). NHIP transcript levels also increased after exposure to $\mathrm{CoCl}_{2}$ specifically in differentiated, but not undifferentiated LUHMES cells (Mann-Whitney-Wilcoxon, $p$ value $=0.009$, differentiated LUHMES treated with $100 \mathrm{nM} \mathrm{CoCl}_{2} n=9$, differentiated LUHMES treated with vehicle $n=9$, effect size $=1.56$, Fig. 2e). Following removal of hypoxia, NHIP transcript levels returned to untreated levels, demonstrating the transience of the response (Additional File 3: Fig. S16). Among the tested human cell lines, embryonic kidney origin HEK293T cells had the lowest endogenous NHIP transcript levels (Fig. 2c). Since response to hypoxia is a developmental signal regulating cell proliferation in embryos [42], we experimentally tested this hypothesis by transiently transfecting HEK293T cells with either a plasmid encoding NHIP with a dual GFP-Puromycin selection cassette or a control vector control lacking NHIP (plasmid construct shown in Additional File 3: Fig. S17, sequences of vector and peptide are in Additional File 12: Table S11). A significantly shortened doubling time was observed in response to NHIP overexpression compared to control cells $(20.23 \mathrm{~h}$ vs. $24.91 \mathrm{~h}$ ) (Hour 142, Mann-Whitney-Wilcoxon, $p$ value $=$ 0.045, NHIP overexpression cells $n=3$, control cells $n=3$, effect size $=3.10$; Hour 166, Mann-Whitney-Wilcoxon, $p$ value $=0.0009$, NHIP overexpression cells $n=3$, control cells $n=3$, effect size $=7.80$, Fig. 2f, Additional File 3: Fig. S18). These results demonstrate that NHIP is a hypoxia-inducible gene in neurons that regulates cell proliferation in an embryonic cell line with low endogenous expression.

To examine whether NHIP encoded a protein, we identified a 20 -amino acid (aa) putative peptide containing a Kozak sequence and tested the existence of the peptide by designing the NHIP peptide-eGFP vector so that the peptide sequence would be in frame with ATG-less GFP transfected in HEK293T cells (Fig. 2g, sequences are in 
Additional File 12: Table S11). The presence of both transfection control (red, mCherry) and reporter (green, eGFP) confirmed the existence of the 20 aa NHIP peptide (Fig. 2g). The NHIP encoded peptide sequence was confirmed using mass spectrometry after pulldown with anti-GFP antibody (Additional File 3: Fig. S19). A search of human protein databases demonstrated that the NHIP peptide partially overlapped protein sequences within BRCA2 and CHD4 (alignments are shown in Additional File 3: Fig. S20, blastp results are in Additional File 13: Table S12). Lastly, using a custom antibody against the NHIP encoded peptide, immunostaining was performed on sections of human postmortem prefrontal cortex, demonstrating nuclear staining in a subset of neuronal nuclei (Fig. 2h). Together, these results demonstrate the existence of a nuclear peptide encoded by NHIP.

\section{A common genetic structural variant at $22 \mathrm{q} 13.33$ is associated with reduced placental DNA methylation, reduced NHIP expression, and increased ASD risk}

To examine genetic factors associated with 22q13.33 methylation levels and polymorphic expression of NHIP in ASD, we tested the association between 22q13.33 block DNA methylation levels and common variants from individual-matched whole genome sequencing (WGS), including SNPs, insertions or deletions (indels), copy number variations (CNVs), and SVs. Methylation levels in five out of 12 ASD DMRs within 22q13.33 were significantly associated with common SNPs located inside the DMRs (linear regression, $p$ values in Additional File 3: Fig. S21). A 1674 bp SV insertion (chr22: 49029657, hg38) was identified 15,013 bp upstream of the start of the 22q13.33 co-methylated block (Fig. 3a, genotypes and demographics of each subject are in Additional File 14: Table S13) with which DNA methylation levels of all 12 22q13.33 ASD DMRs were significantly associated (linear regression, $p$ values are in Fig. 3b). In the MARBLES cohort, this SV insertion was identified in significantly more ASD than TD samples (chi-square test, ASD $n=41$, TD $n$ $=37, p$ value $=0.045$ ) (Additional File 3: Fig. S22). Placenta samples with the 22q13.33 insertion from ASD, but not TD, showed significantly lower methylation levels (methylation vs diagnosis: Mann-Whitney-Wilcoxon, $p$ value $=0.008$, ASD $n=41$, TD $n=37$, effect size $=-0.645$; methylation vs genotype: Mann-Whitney-Wilcoxon, $p$ value $=0.004$, Y $n=$ $29, \mathrm{~N} n=49$, effect size $=-0.644$; in ASD samples: methylation vs genotype: MannWhitney-Wilcoxon, $p$ value $=0.005, \mathrm{Y} n=20, \mathrm{~N} n=21$, effect size $=-0.878$; in TD samples: methylation vs genotype Mann-Whitney-Wilcoxon, $p$ value $=0.847, \mathrm{Y} n=9, \mathrm{~N} n=$ 28, effect size $=-0.173$; Fig. 3c). While not present in the reference genome, the 22q13.33 insertion was also identified as a structural variant identified from PacBio assembly data of the human CHM1 complete hydatidiform cell line (CHM1_chr22-49029645-INS-1673 contig [43] and NCBI GenBank ID QPKN01007947.1 [44] Additional File 3: Fig. S23). We also confirmed the WGS identification of the SV using PCR genotyping primer sets and provided allelic genotypes (genotypes are in Additional File 14: Table S13, example of PCR products is in Additional File 3: Fig. S24). The insertion sequence showed high similarity with retrotransposon elements, including SVA and Alu (Additional File 3: Fig. S25). This 22q13.33 SV also corresponded to INS_22_115103 in Genome Aggregation Database (gnomAD) which showed an average allele frequency of 0.7 and only small deviation (10\% or less) based on ancestry or sex (Additional File 3: Fig. S26) [45, 46].

Since the 22q13.33 block exhibited lower methylation in ASD compared to TD placental samples from the MARBLES cohort, we chose to evaluate the relationship 


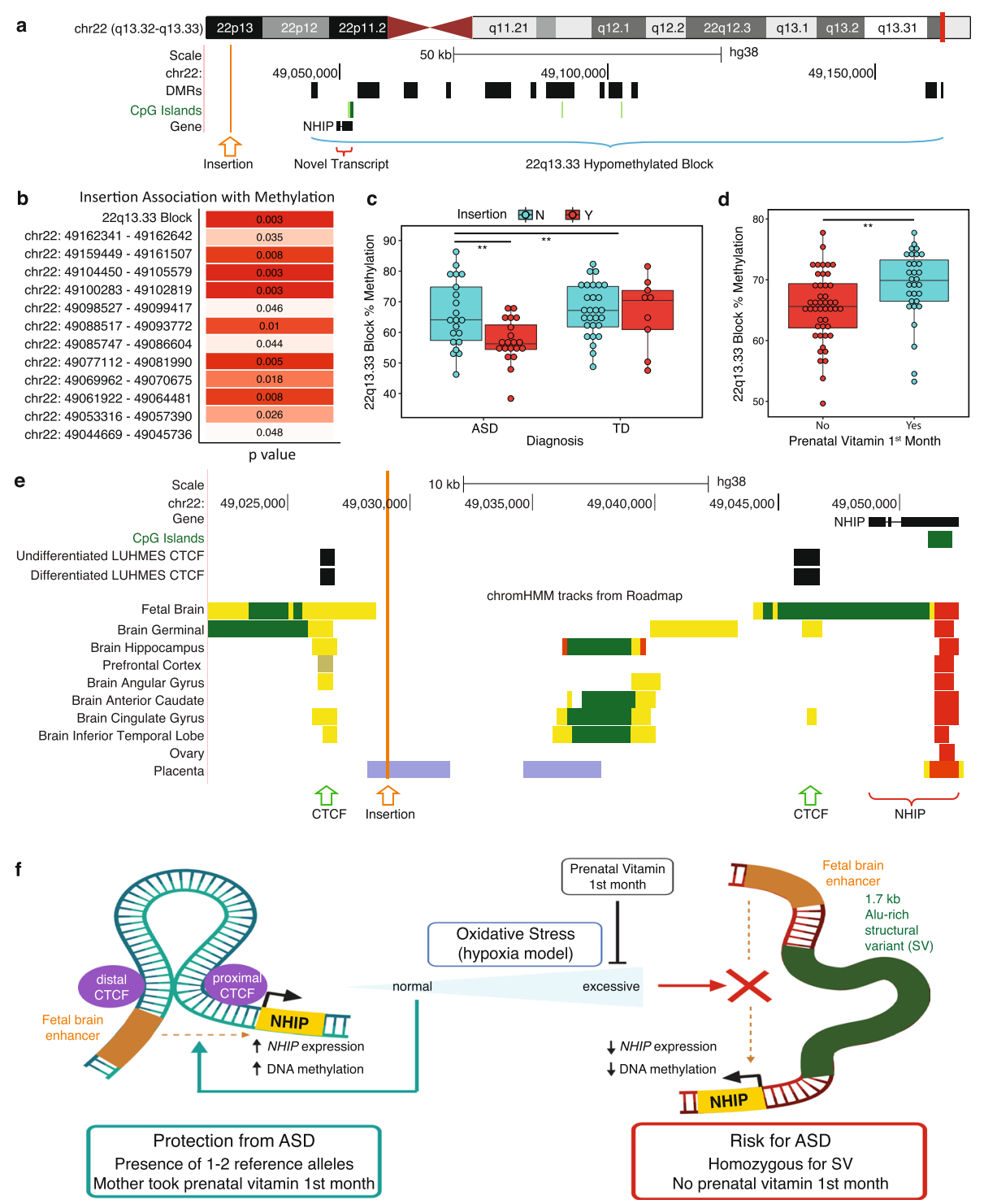

Fig. 3 (See legend on next page.) 
(See figure on previous page.)

Fig. 3 A common genetic structural variant is significantly associated with 22q13.33 DNA methylation and ASD. A Insertion location (orange) relative to the 22q13.33 hypomethylated block (blue), and the novel transcript, NHIP (red) in the UCSC genome browser. The 22q13.33 co-methylated block was 117,974 bp in length (blue). NHIP TSS was located 7881 bp downstream from the start of the 22q13.33 hypomethylated block. The insertion (not in the reference genome) is 15,013 bp upstream from the start of the 22q13.33 hypomethylated block. B The association matrix shows ANOVA $p$ values for the comparison of the insertion genotype (homozygous for insertion versus not) with smoothed methylation levels within each of 12 DMRs located in the 22q13.33 hypomethylated block from the discovery group (ASD $n=41, T D n=37$ ). C Association was tested between insertion genotype ( $Y$, homozygous for insertion; N, not) and 22q13.33 comethylated block methylation levels (discovery group, ASD $n=41$, TD $n=37$ ). ASD showed significantly lower DNA methylation levels compared to TD placenta samples within the entire 22q13.33 co-methylated block (Mann-Whitney-Wilcoxon, $p$ value $=0.008$, ASD $n=41$, TD $n=37$, effect size $=-0.645$ ). Samples homozygous for the insertion had significantly lower methylation than those not having insertion on one or both alleles (Mann-Whitney-Wilcoxon, $p$ value $=0.004, Y n=29, N n=49$, effect size $=-0.644$ ). When broken down by diagnosis, samples with insertion had significantly lower methylation specifically in ASD samples (Mann-Whitney-Wilcoxon, $p$ value $=0.005, Y n=20, N n=21$, effect size $=-0.878$ ), not TD samples (Mann-Whitney-Wilcoxon, $p$ value $=0.847, \mathrm{Y} n=9, \mathrm{~N} n=28$, effect size $=-0.173$ ). D Periconceptional prenatal vitamin use was a significant modifier of 22q13.33 block methylation in placenta (discovery group, ASD $n=41$, TD $n=37$ ). Lower percent methylation at the 22q13.33 co-methylated block was significantly associated with not taking prenatal vitamins during the first month of pregnancy (Mann-Whitney-Wilcoxon, $p$ value $=0.001$ ), which was in the same direction as ASD risk. $\mathbf{E}$ UCSC genome browser map shows the insertion location (orange vertical line) relative to two adjacent CTCF sites (green arrows) and NHIP. Both undifferentiated and differentiated LUHMES cells have both CTCF sites, consistent with them being homozygous for the reference sequence. Additional brain tracks show the variability of the upstream CTCF site between human samples. ChromHMM tracks were derived from fetal brain, multiple brain regions, ovary, and placenta. Red, active promoter; yellow, active enhancer; green; active transcriptional elongation; purple, bivalent poised chromatin. F Working model to explain ASD risk associated with SV homozygosity. Illustrations created with BioRender.com

between prenatal vitamin use during the first month of pregnancy, previously shown to be associated with decreased ASD risk in this cohort [47], in the general population [48, 49], and in other high-familial risk cohorts [50], in the context of ASD risk associated with the insertion. Along with other vitamins and minerals, prenatal vitamins contain high folic acid to meet the increased needs during pregnancy, and could be an important source of methyl donors. There was a significant positive association with prenatal vitamins use in the first month and methylation level at the 22q13.33 block, in the protective direction (Mann-Whitney-Wilcoxon, $p$ value $=0.001$, took prenatal vita$\min n=31$, did not take prenatal vitamin $n=46$, effect size $=0.477$ ) (Fig. 3d). When samples were stratified by 22q13.33 insertion genotype, prenatal vitamin use during the first month of pregnancy showed a nominally significant protective effect among individuals with the insertion, although we acknowledge that the sample size is small (within samples homozygous for the insertion: in samples from mothers who took prenatal vitamin during the first month of pregnancy, Mann-Whitney-Wilcoxon, $p$ value = 0.046 , ASD $n=3$, TD $n=4$, effect size $=-2.09$; Additional File 3: Fig. S27). Unlike the 22q13.33 insertion, the GWAS-based PRS [8] calculated for the MARBLES cohort was not significantly different between diagnostic groups or associated with 22q13.33 block methylation by ANOVA in the MARBLES discovery cohort (Additional File 15: Table S14). Together, these results are consistent with the hypothesis that ASD risk associated with the 22q13.33 SV and co-methylated block is distinguishable from polygenic ASD risk and tempered by a common nutrient intervention with evidence for an association with ASD protection. 
Since SVs have been previously implicated in altering chromatin loops regulating promoter-enhancer interactions [51], we hypothesized that this $1.7 \mathrm{~kb}$ insertion may be located within an enhancer-promoter loop relevant to fetal brain. Using the recent EpiMap database of chromatin states across multiple humans and tissue types [52], we identified two CTCF sites flanking the SV insertion (Fig. 3e). ChromHMM maps [35] demonstrate a fetal brain enhancer that aligns with the distal CTCF binding site. The proximal CTCF site is adjacent to the NHIP TSS, which ChromHMM predicts as an active promoter in brain, ovary, and placenta. These two CTCF binding sites were inside a large $\sim 2 \mathrm{Mb}$ topologically associated domain (TAD) spanning from the $48.5 \mathrm{Mb}$ position to the telomere of 22q (Additional File 3: Fig. S28) [53]. Together, these results suggest a model whereby the SV insertion allele could disrupt the fetal brain enhancerpromoter interaction within a large telomeric TAD, thereby reducing the responsiveness of NHIP expression to neuronal differentiation and excessive oxidative stress (Fig. 3f). Early pregnancy prenatal vitamin use is expected to counteract the effects of oxidative stress through provision of dietary methyl groups, thereby increasing DNA methylation at the NHIP locus in individuals homozygous for the 22q13.33 insertion.

\section{NHIP expression is reduced in ASD brain and associated with the regulation of genes enriched for synaptic functions and ASD risk}

We then tested the hypothesis that the 22q13.33 insertion was associated with NHIP expression in ASD versus TD postmortem brain samples that were matched for age, sex, and race/ethnicity (Additional File 16: Table S15). Similar to the MARBLES cohort of placenta samples, the 22q13.33 insertion showed a significantly higher frequency in ASD compared with TD in a necessarily small group of 58 cortical samples acquired from postmortem brain banks (chi-square test, ASD $n=27$, TD $n=30, p$ value $=$ 0.023; Additional File 3: Fig. S29). RNA-seq was performed on a subset of 20 cortical samples representing all three SV insertion genotypes, matched for age, sex, and race/ ethnicity between ASD and TD. Brain samples homozygous for the 22q13.33 insertion (Y) exhibited lower NHIP levels compared to those with one or no insertion alleles $(\mathrm{N})$ specifically in ASD, but not in TD samples (Fig. 4a).

We then performed a genome-wide analysis of transcript levels associated with variable NHIP transcript levels in brain samples as a continuous response variable. In total, 851 genes passed FDR significance for NHIP association, including 195 positively and 656 negatively associated (Fig. 4b, gene list is in Additional File 17: Table S16). After adjustment for sex, age, brain region, and postmortem interval (PMI), 534 genes passed FDR significance for NHIP association, of which 445 overlapped with those identified without covariate adjustments, including 166 positively and 368 negatively associated with NHIP (Additional File 3: Fig. S30, gene list is in Additional File 18: Table S17). Downregulated genes included ASD candidate genes such as CHD8 [54], and a gene previously implicated in ASD from placenta, IRS2 [21] (unadjusted gene list in Additional File 17: Table S16, adjusted gene list in Additional File 18: Table S17). Gene ontology (GO) enrichment analysis of NHIP-associated genes (covariate adjusted) revealed 252 terms significant by permutation test (Fig. 4c, FDR-adjusted $p$ values in Additional File 19: Table S18). Regulation of nervous system development, including gliogenesis, synaptic vesicle transport, and dendritic spine development, and response 


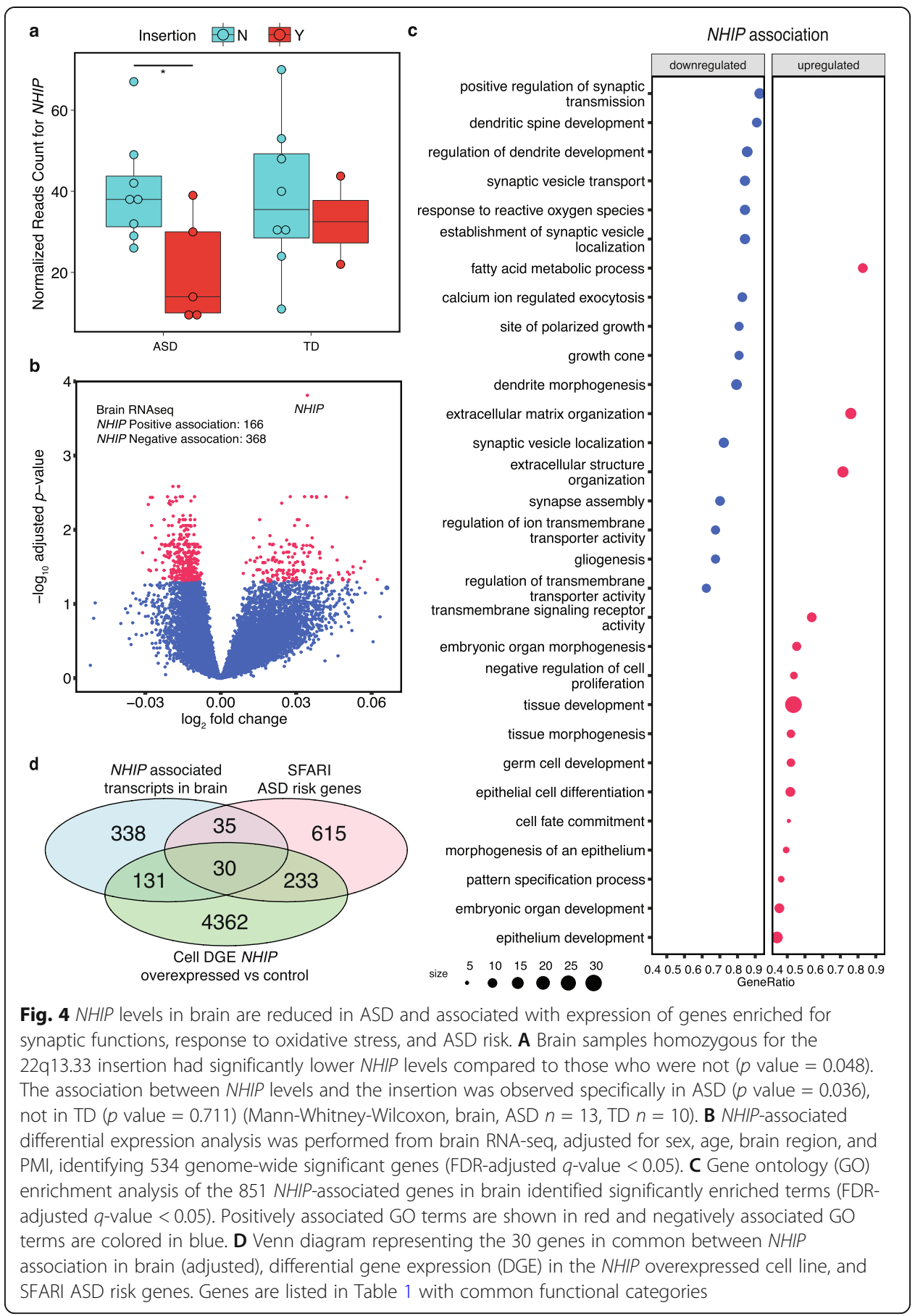

to oxidative stress were negatively associated with NHIP transcript levels (Fig. 4c, GO term analyses are in Additional File 19: Table S18). GO term functions related to the dendritic spine, synapse assembly, and response to reactive oxygen formed a functional module of genes negatively associated with NHIP levels (Additional File 3: Fig. S31, Additional File 19: Table S18). In contrast, transcripts positively associated with NHIP levels were enriched for distinct functions in fatty acid metabolism and embryonic organ development. To further examine the relevance of NHIP expression to ASD etiology, we overlapped brain $N H I P$-associated transcripts with SFARI ASD risk genes 
and observed a significant overlap of 65 genes (Fisher's exact test, $p$ value $=1.572 \mathrm{E}-13$, $\mathrm{OR}=3.131$, Additional File 3: Fig. S32, gene list is in Additional File 20: Table S19). The 65 genes in common were significantly enriched for 14 GO terms, including nervous system development, synapse, chromatin organization, and neurogenesis (Additional File 3: Fig. S33, FDR-adjusted $p$ values in Additional File 21: Table S20), demonstrating associations of NHIP levels with functionally relevant gene pathways in brain and ASD.

\section{Overexpression of NHIP in HEK293T cells results in large-scale transcriptional changes to genes relevant to brain and ASD risk}

To experimentally model the transcriptional impact of NHIP induction, RNA-seq and differential expression analyses were performed on HEK293T cells transiently transfected with NHIP or vector control. We identified 4756 differentially expressed genes (DEG) with genome-wide significance (FDR-adjusted $p$ value $<0.05$ ). NHIP overexpression increased expression of 1490 genes and decreased expression of 3266 genes (Additional File 3: Fig. S34, gene list is in Additional File 22: Table S21). Genes decreased with NHIP expression included the downstream flanking gene BRD1, as well as IRS2, CHD8, and DLL1 (Additional File 3: Fig. S35, Additional File 22: Table S21). NHIP overexpression and reduced $B R D 1$ in overexpression cell lines were confirmed with RT-PCR (Additional File 3: Fig. S36). Genes differentially expressed with NHIP overexpression were enriched for GO terms associated with noncoding RNA processing, histone modification, placental development, cell cycle, and p53 binding (Additional File 3: Fig. S37, GO term enrichment statistics are in Additional File 23: Table S22), consistent with the proliferation phenotype (Fig. 2f). KEGG gene set enrichment analysis [55] showed enrichment for brain disorders, including Parkinson's, Alzheimer's, and Huntington's diseases and metabolism, such as fatty acid metabolism and drug metabolism (Additional File 3: Fig. S38, KEGG enrichment statistics are in Additional File 24: Table S23), further demonstrating the relevance of NHIP regulated genes to brain functions.

In a comparison of in vivo and in vitro RNA-seq analyses, 161 genes overlapped between those differentially expressed in response to experimental NHIP overexpression and those associated with NHIP transcript levels in human brain, which was significant compared to all expressed genes $(\geq 1$ read in any sample, Fisher's exact test, $p$ value $=$ $6.946 \mathrm{E}-5$, OR $=1.482$; or $\geq 1$ read per sample in both tissues, Fisher's exact test, $p$ value $=8.348 \mathrm{E}-4$, OR = 1.386; Additional File 3: Fig. S39, Additional File 25: Table S24). Genes negatively associated with NHIP levels in vitro and in vivo were enriched for functions in synapse, chromatin, and regulation of nervous system development (Additional File 3: Fig. S40, gene list is in Additional File 26: Table S25). Similarly, 20 GO terms overlapped between in vivo and in vitro RNA-seq analyses including regulation of translation, peptide biosynthetic process, and rhythmic process, which was significant compared to all GO terms (Fisher's exact test, $p$ value $=2.24 \mathrm{E}-5$, OR $=0.137$, Additional File 27: Table S26). Furthermore, genes differentially expressed with NHIP overexpression also showed a significant overlap of 263 genes with ASD risk genes from the SFARI database compared to all expressed genes (Fisher's exact test, $p$ value $=$ $2.752 \mathrm{E}-05, \mathrm{OR}=1.379$ ) and were enriched for functions in central nervous system development, synaptic signaling, and response to oxygen levels (Additional File 3: Fig. 
S41-S42, FDR-adjusted $p$ values are in Additional Files 28-29: Tables S27-S28). There were 30 genes in common among ASD risk, NHIP association in brain, and NHIP overexpression, including BRD4, SETD5, ARID1B, EP300, and FOXG1 (Fig. 4d, Table 1, FDRadjusted $p$ values are in Additional File 30: Table S29). Genes common to ASD risk, NHIP association in brain, and NHIP overexpression were enriched for chromatin organization, regulation of transcription by RNA polymerase II, histone modification, neurogenesis, and rhythmic processes (Table 1, Additional File 3: Fig. S43, GO term enrichment statistics are in Additional File 31: Table S30). Together, these results demonstrate that NHIP is a novel regulatory gene with functions relevant to known ASD risk factors.

\section{Discussion}

This study has taken the innovative approach of utilizing placental tissue from a highrisk prospective pregnancy cohort with multi-omic assays to discover a novel ASD risk

Table 1 Functional categories of genes showing adjusted NHIP-associated expression in human cortex, differential expression in NHIP overexpressing cells, and known ASD risk (from Fig. 4d overlap)

\begin{tabular}{|c|c|c|c|c|c|c|c|}
\hline \multirow[b]{2}{*}{ Genes } & \multicolumn{6}{|l|}{ GO Terms } & \multirow[b]{2}{*}{ Count } \\
\hline & $\begin{array}{l}\text { Chromatin } \\
\text { organization }\end{array}$ & $\begin{array}{l}\text { Regulation of } \\
\text { transcription by } \\
\text { RNA polymerase II }\end{array}$ & $\begin{array}{l}\text { Histone } \\
\text { modification }\end{array}$ & $\begin{array}{l}\text { Rhythmic } \\
\text { process }\end{array}$ & $\begin{array}{l}\text { Dendritic } \\
\text { spine }\end{array}$ & $\begin{array}{l}\text { Regulation } \\
\text { of } \\
\text { biosynthetic } \\
\text { process }\end{array}$ & \\
\hline$A R I D 1 B$ & $x$ & $x$ & & & & x & 3 \\
\hline ASHIL & $x$ & $x$ & $x$ & & & x & 4 \\
\hline BRD4 & $x$ & $x$ & $x$ & & & x & 4 \\
\hline CREBBP & $x$ & $x$ & $x$ & $x$ & & x & 5 \\
\hline EP300 & $x$ & $x$ & $x$ & $x$ & & x & 5 \\
\hline HNRNPU & $x$ & $x$ & & $x$ & & x & 4 \\
\hline HUWE1 & $x$ & & $x$ & $x$ & & & 3 \\
\hline JMJDIC & $x$ & & $x$ & & & x & 3 \\
\hline KMT2A & $x$ & $x$ & $x$ & $x$ & & x & 5 \\
\hline PRKCA & $x$ & & $x$ & & & & 2 \\
\hline RERE & $x$ & $x$ & & & & x & 3 \\
\hline SETD5 & $x$ & & $x$ & & & & 2 \\
\hline SMARCA2 & $x$ & $x$ & & & & x & 3 \\
\hline$A D N P$ & & $x$ & & $x$ & & x & 3 \\
\hline CUX1 & & $x$ & & & & x & 2 \\
\hline FOXG1 & & $x$ & & & & x & 2 \\
\hline GRIN1 & & $x$ & & & $x$ & x & 3 \\
\hline NR1D1 & & $x$ & & $x$ & $x$ & x & 4 \\
\hline NR2F1 & & $x$ & & & & $x$ & 2 \\
\hline POGZ & & $x$ & & & & x & 2 \\
\hline ZNF292 & & $x$ & & & & $x$ & 2 \\
\hline $\begin{array}{l}\text { ARHG } \\
\text { AP32 }\end{array}$ & & & & & $x$ & & 1 \\
\hline CPEBA & & & & & $x$ & $x$ & 2 \\
\hline$\angle R R C 4$ & & & & & $x$ & & 1 \\
\hline TNRC6B & & & & & & $x$ & 1 \\
\hline
\end{tabular}


gene locus that integrates responsiveness to oxidative stress with inheritance of a common structural variant. Given the distinctive DNA methylation landscape of the placenta characterized by PMDs and higher gene body methylation over expressed genes $[14,15,56]$, using unbiased WGBS as a tool enabled the discovery of a novel gene associated with ASD that had been missed by standard genetic and epigenetic array-based approaches. The 22q13.33 co-methylated block identified in this study was previously identified by WGBS as a correlated region of increased methylation variance (CoRSIV) $[24,25]$ as well as a region of increased SV [30] in the human genome. We confirmed the hypothesis that CoRSIV and SV locations overlap more frequently than expected at random (Additional File 3: Fig. S44). Although this 22q13.33 region has not been previously associated with ASD risk, the neighboring distal long arm of 22q13.3 harbors multiple genes implicated in neuropsychiatric disorders, including ASD, intellectual disability, schizophrenia, and bipolar disease [57-59]. SHANK3, which encodes a postsynaptic protein required for maturation of glutamatergic synapses [60], is $1.5 \mathrm{Mb}$ telomeric from the 22q13.33 hypomethylated block identified in this study. Rare SHANK3 mutations are noted in ASD [61], and large structural variations including SHANK3 are observed in rare ASD children [57]. In addition, 22q13.33, 22q13.32, and 22q13.31 are disease-associated hotspot regions in ASD [33]. While these highly polymorphic regions of the genome have the potential to contain regulatory genes such as NHIP, as well as primate-specific sequences relevant to brain development [62], they are often excluded from the design of array-based platforms because of their complexities. The NHIP locus is sparsely covered by probes in the most current genetic and epigenetic array designs (Additional File 3: Fig. S14), a likely explanation for why it was not identified by prior ASD studies. In contrast, sequencing-based approaches, such as the integrated WGS and WGBS approach employed here, are a promising alternative for disease association testing.

Placenta is an often misunderstood and overlooked tissue, despite its importance in regulating and thereby reflecting events critical to brain development in utero. Placenta regulates metabolism and provides steroid hormones as well as neurotransmitters critical for the developing brain $[63,64]$. Additionally, placenta regulates oxygen supply, as it consumes $40-60 \%$ of the body's oxygen, and hypoxia metabolic adaptation regulates trophoblast cell fate decisions [65, 66]. Oxygen tension can also modulate extravillous trophoblast proliferation, differentiation, and invasion [67], all important for successful implantation and placentation, which can all impact brain development and ASD risk [68-70].

We have demonstrated that NHIP is a primate-specific, variably expressed gene responsive to hypoxia in human placenta and brain tissues. The variability in NHIP transcript levels was influenced by both non-genetic and genetic factors. First, NHIP was induced with neuronal differentiation, but also with hypoxia and oxidative stress. Interestingly, the responsiveness of NHIP expression as well as oxidative stress was specific to differentiated neurons but not seen in the undifferentiated state. Oxidative stress is a common convergent mechanism that occurs in normal neurodevelopment but can be excessive in cases of many environmental exposures associated with in ASD, including air pollution [71] and pesticides [72]. Second, prenatal vitamin use in the first month of pregnancy provides essential methyl donors to the one-carbon metabolism pathway $[49,73]$ that may counteract excessive oxidative stress, a prediction consistent with the 
elevated methylation over the 22q13.33 block in placentas from pregnancies with first month prenatal vitamin use. Third, common genetic variants were also associated with 22q13.33 methylation levels. While we identified 12 SNPs within the 22q13.33 comethylated block that were significantly associated with methylation, the strongest genetic factor was a $1.7 \mathrm{~kb}$ insertion with a high allele frequency in all ethnicities. Homozygosity for this 22q13.33 insertion was a better predictor of ASD risk than GWAS-based PRS in this mixed ancestry cohort. 22q13.33 SV homozygosity was also strongly associated with hypomethylation of this locus and reduced expression of NHIP in ASD compared to TD placenta and brain samples.

Large insertions such as the 22q13.33 SV that occur outside of coding regions can still modify gene expression through alterations in promoter-enhancer loop size. The NHIP promoter shows differences in active chromatin marks between individuals and is associated with two CTCF binding sites that apparently anchor an intra-TAD loop between the promoter and a distal fetal brain enhancer. These results suggest a model by which the presence of at least one copy of the reference allele without the insertion would allow NHIP to be induced during neurodevelopment and hypoxia, thereby protect the developing brain through its regulation of downstream regulatory gene pathways (Fig. 3f). Homozygosity for the 22q13.33 SV allele is associated with lower NHIP expression and less protection, likely because the enhancer-promoter loop forms less efficiently because of the $>15 \%$ increased size of the loop. For the minority of TD children who were also homozygous for the 22q13.33 SV, the use of prenatal vitamins that can reduce the consequences of oxidative stress might have been one source of associated protection from risk, although other genetic and environmental factors not investigated may also be involved.

There are several limitations to this study. Because of the relatively small sample size of placentas that came from the only two available ASD-enriched longitudinal studies in the USA (MARBLES and EARLI), the association between 22q13.33 epigenetic and genetic variation with ASD should be replicated in future birth cohorts. Further, the postmortem brain samples used for RNA-seq also constituted relatively small sample sizes, although comparable to other postmortem brain studies in ASD [74-76]. Because the NHIP-associated insertion is only detectable in whole genome sequencing studies, not whole exome or SNP arrays, we were limited in performing a larger population analysis of the allele frequencies of the insertion in ASD. In spite of these necessary inherent limitations, this study used an unconventional approach to identify a new gene locus of potential relevance to ASD etiology that will need confirmation by future investigations.

\section{Conclusions}

Much of the human genome is still under-represented in genome- and epigenomewide association studies with complex diseases such as autism, resulting in an incomplete understanding of heredity, risk, and resilience. Using the novel approach of combining WGBS with WGS in placenta and cord blood samples from two prospective ASD enriched risk cohorts, this study identified a novel regulatory gene NHIP expressed in response to hypoxia in placenta and neurons, but modified by a novel structural variant associated with increased ASD risk. These results are expected to be important in understanding gene by environmental interactions in the developing brain 
and as potential biomarkers of ASD risk at birth, prior to the development of symptoms.

\section{Methods}

\section{Sample population and diagnostic classification}

The Markers of Autism Risk in Babies - Learning Early Signs (MARBLES) study [77] recruited mothers with at least one child that had been diagnosed with ASD and who were pregnant or planning another pregnancy in Northern California, primarily through lists provided by the California Department of Development Services [21, 7779]. The following criteria were required for MARBLES study's enrollment: the prospective child has at least one first or second degree relative diagnosed with ASD; the mother is at least 18 years old; the mother is pregnant or planning for a pregnancy; the mother speaks, reads, and understands English proficiently enough in order to complete the protocol; and the mother lives within a 2.5-h drive distance of Davis/Sacramento region. Demographic, diet, and medical information were collected by prospective telephone interviews or questionnaires throughout the pregnancy. For this analysis, a discovery set of 46 placentae from children subsequently diagnosed with ASD and 46 placentae from children subsequently found to have typical neurodevelopment (TD) was sequenced. An internal WGBS replication group included 65 additional MARBLES placenta samples (ASD $n=21$, Non-TD $n=13$, TD $n=31$ ). Finally, whole genome sequence data were available on 41 ASD and 37 TD MARBLES children, which were used for SNP and SV analyses to characterize WGBS findings.

The Early Autism Risk Longitudinal Investigation (EARLI) study recruited pregnant mothers who already have a child diagnosed with ASD and has been described in detail previously [80]. EARLI families were recruited from four sites (Drexel/Children's Hospital of Philadelphia, Johns Hopkins/Kennedy Krieger Institute, Kaiser Permanente Northern California, and University of California, Davis) across three US regions (Southeast Pennsylvania, Northeast Maryland, and Northern California). Enrollment criteria for EARLI were as follows: having a biological child diagnosed with ASD; communicating fluently in English or Spanish; being 18 years or older; living within a 2-h drive distance from the study site; and being less than 29 weeks pregnant. For replication analysis of the initial MARBLES WGBS findings, 47 placenta samples (ASD $n=16$, TD $n=31$ ) were available from the EARLI study, with details described previously [81].

In both MARBLES and EARLI studies, the subsequent child diagnosis was clinically assessed by trained, professional examiners at 36 months using standardized instruments including the Autism Diagnostic Observation Schedule (ADOS) [82], Autism Diagnostic Interview - Revised (ADI-R) [83], and Mullen Scales of Early Learning (MSEL) [84]. Based on a previously published algorithm, children were classified into three outcome groups: ASD, TD, and Non-TD [47, 85, 86]. Children with ASD had scores over the ADOS cutoff and fit ASD DSM-5 criteria. Children with TD had all MSEL scores within 2 standard deviations (SD) and no more than one MSEL subscale 1.5 SD below the normative mean together with scores on the ADOS at least three points lower than the ASD cutoff. Children with Non-TD did not meet ASD or TD criteria, but had elevated ADOS scores and low MSEL scores, defined as two or more 
MSEL subscales with more than 1.5 SD below the normative mean or at least one MSEL subscale more than 2 SD below the normative mean.

\section{Whole genome bisulfite sequencing (WGBS) library preparation}

The placental samples were frozen within $4 \mathrm{~h}$ after birth. DNA was extracted from placenta tissue with the Gentra Puregene kit (Qiagen, Hilden, Germany) and quantified with the Qubit DNA Assay Kit (Thermo Fisher Scientific, Waltham, MA, USA). The discovery group included 92 samples (ASD $n=46$, TD $n=46$ ) from the MARBLES study that were used for both WGBS and WGS. For WGBS, DNA was bisulfite converted with the EZ DNA Methylation Lightning kit (Zymo, Irvine, CA, USA). WGBS libraries were prepared from bisulfite-converted DNA using the TruSeq DNA Methylation kit (Illumina, San Diego, CA, USA) with indexed PCR primers and a 14 cycle PCR program. Libraries were sequenced at 2 per lane with $150 \mathrm{bp}$ paired-end reads in Illumina HiSeq X (San Diego, CA, USA) by Novogene (Sacramento, CA, USA). The external replication group included WGBS data from 47 samples (ASD $n=16$, TD $n=31$ ) from the EARLI study, with details described previously [81]. The specificity replication group included 65 samples (ASD $n=21$, Non-TD $n=13$, TD $n=31$ ) from the MARBLES study. DNA were sonicated to $~ 350$ bp using a Covaris E220 (Woburn, MA, USA). Sonicated and size selected DNA was bisulfite converted using the EZ DNA Methylation Lightning kit (Zymo, Irvine, CA, USA). WGBS libraries were prepared using Accel-NGS Methyl-Seq DNA library kit (Swift Biosciences, Ann Arbor, MI, USA) with indexed PCR primers and a 12-cycle PCR program. Libraries were pooled and sequenced on 2 lanes with $150 \mathrm{bp}$ paired-end reads of Illumina NovaSeq 6000 S4 (San Diego, CA, USA) by the DNA Tech Core at University of California, Davis (Davis, CA, USA).

\section{WGBS alignment and quality control}

Raw sequencing files were preprocessed, aligned to the human reference genome, and converted to CpG methylation count matrices with the default parameters in CpG_Me [87-89]. Reads were trimmed to remove adapters and methylation bias on both $5^{\prime}$ and 3' end. After trimming, reads were aligned to human reference genome hg38 and filtered for PCR duplicates. Cytosine methylation reports were generated using all covered CpG sites. Quality control was examined for each sample and independently between the three sample cohorts because of the different sequencing platforms used. Libraries with $\mathrm{CHH}$ methylation greater than $2 \%$ were excluded as incomplete bisulfite conversion. The CpG_Me workflow incorporates Trim Galore, Bismark, Bowtie2, SAMtools, and MultiQC [88, 90-93].

\section{Global methylation and principal component analysis (PCA)}

DNA methylation at 20-kb windows sliding across the genome was extracted using the getMeth function in the bsseq $R$ package [94, 95]. Percent methylation for each sample at each window was calculated using the average methylation value from the window. Correlations between DMRs were calculated using Pearson's correlation coefficient. Principal component analysis (PCA) was performed using the prcomp function in the 
stats $R$ package and visualized using ggbiplot [96]. The ellipses for each group were illustrated as the $95 \%$ confidence limit.

\section{Methylation array analysis and cell type estimation}

The same 92 placenta DNA sample aliquots in the discovery group (ASD $n=46$, TD $n$ = 46) were used for DNA methylation array analysis. DNA was treated and cleaned with the EZ DNA methylation gold kit (Zymo, Irvine, CA, USA). Samples were assayed on the Infinium MethylationEPIC array (Illumina, San Diego, CA, USA) at John Hopkins University CIDR (Baltimore, MD, USA). Raw image files were analyzed using the minfi R package [97]. Data were corrected for background and dye bias with the normal-exponential by out-of-band probe (noob) method [98]. Cell type composition of placenta (trophoblast cells, stromal cells, Hofbauer cells, endothelial cells, and nucleated red blood cells) was estimated from DNA methylation array in the discovery group, WGBS methylation in the external replication and specificity replication group using a sorted placenta cell reference by PlaNET [99].

\section{Detection of DMRs}

DMRs were identified between ASD and TD in the discovery group through DMRichR, with 100 permutations and adjustments for sex and cell types [87, 100]. DMRichR utilized the dmrseq and bsseq algorithms to process methylation levels from a CpG count matrix to identify DMRs $[94,101]$. The DMR analysis approach used a smoothing and weighting algorithm that weights CpGs based on coverage. CpGs in physical proximity with similar methylation values were grouped into candidate background regions to estimate region statistics. In permutation testing, DMR percent methylation values were randomly shuffled between diagnosis groups and pooled together to form an approximate null distribution using bsseq [94]. The empirical $p$ value was calculated by comparing the observed test to the entire null distribution from all permutation tests to identify significant DMRs. Further correction for genome-wide significance used a FDR of 0.05. Repeating the DMR permutation test with 90 instead of 100 permutations did not change the number of DMRs detected (134 significant by empirical $p$ value, 1 after FDR correction) (Additional File 4: Table S3). Individual smoothed methylation levels and chr22q block methylation levels were obtained using the bsseq R package [94]. Genes were assigned to DMRs using the Genomic Regions Enrichment of Annotation Tool (GREAT) tool with the default association settings ( $5 \mathrm{~kb}$ upstream, $1 \mathrm{~kb}$ downstream, and $1000 \mathrm{~kb}$ max extension) [102]. The distances (kb) were calculated from DMRs to the transcription start site (TSS) of the GREAT assigned genes. Gene Ontology (GO) enrichment analysis for DMRs, hypermethylation DMRs, and hypomethylation DMRs relative to background regions was done using GREAT [102]. Significant terms were called with FDR-corrected $p$ values less than 0.05 .

\section{Placenta DMR enrichment analysis}

DMRs were examined for enrichment with chromatin marks compared to the background regions using the LOLA $R$ package with Fisher's exact test followed by FDR correction [103]. Chromatin states were predicted by ChromHMM, which uses a Hidden Markov Model to separate the human genome into 15 functional states based on 
data from the Roadmap Epigenomics Project [35, 104]. Promoter-related states included active TSS (TssA) (red), TSS flank (TssAFlnk) (orange red), bivalent TSS (TssBiv) (Indian Red), and bivalent TSS flank (BivFlnk) (Dark Salmon) states. Enhancer-related states included genic enhancer (EnhG) (Green Yellow), enhancer (Enh) (Yellow), and bivalent enhancer (EnhBiv) (Dark Khaki). CpG island, shore, shelf, and open sea coordinates were obtained from the annotatr $R$ package [105]. Encyclopedia of DNA Elements (ENCODE) datasets were used to extract histone posttranslational modifications (PTMs), including H3K4me1, H3K4me3, H4K9me3, H3K36me3, H3K27me3, and H3K27ac datasets $[37,106]$. Enrichment for known transcription factor binding site motif sequences in DMRs was obtained using Hypergeometric Optimization of Motif EnRichment (HOMER) [107].

\section{Participant whole genome sequencing (WGS) and variant calling}

WGS was performed using cord blood DNA from a subset of the same individuals in the discovery group (ASD $n=41$, TD $n=37$ ). Sequencing libraries were generated using the NEBNest DNA library prep kit (NEB, Ipswich, MA, USA) with 150 bp pairedend reads in Illumina HiSeq X (San Diego, CA, USA) by Novogene (Sacramento, CA, USA) with at least $30 \times$ coverage per sample. Raw read files were mapped to human reference genome hg38 using Burrows-Wheeler Aligner (BWA) with the default settings [108]. SAMtools was utilized to sort the bam files and Picard was used to merge bam files from the same sample and identify duplicate reads [92, 109]. Single-nucleotide polymorphisms (SNPs), and small insertions and deletions (InDels) were called using GATK and variants were annotated using ANNOVAR [110, 111]. Copy number variations (CNVs) longer than 50 bp were identified using control-FREEC and CREST [112, 113]. Structural variant (SV) detection and genotyping, larger than $50 \mathrm{bp}$, were performed using DELLY with the default settings [114]. Our criteria included filtering for minor allele frequency (MAF) $>5 \%$. The association of the DMR taken from individual methylation levels using bsseq [94], and variants by linear regression.

\section{Polygenic risk score (PRS) generation}

A subset of individuals from the discovery group were also genotyped using the Illumina Multi-Ethnic genotyping array ( $\operatorname{ASD} n=31$, TD $n=35$ ). Stringent QC criteria were used on the raw genotypes in order to remove low-quality SNPs and samples [115]. Our criteria included removal of samples with call rates $<98 \%$, sex discrepancy, and relatedness (pi-hat $<0.18$ ) to non-familial samples with filtering out MAF $<5 \%$ using PLINK software [116]. After data cleaning, the imputation pipeline was performed using the University of Michigan Imputation Server [117] with minimac4 software [118] to the 1000G Phase v5 reference panel (hg19) [119, 120]. Phasing was performed using Eagle software [121].

PRS calculation was performed on the imputed genetic data, after applying postimputation filtering $(R$-squared $>0.80)$. PRS was informed by discovery GWAS results from the combined PGC-iPSYCH genome-wide meta-analysis [8] and generated at a range of $p_{\text {discovery }}$ thresholds ( $p_{\text {discovery }}$ threshold range from $1 \times 10^{-8}$ to 1.0). Using PLINK software [116], we removed correlated SNPs and applied from 2 to $>20,000$ effect sizes to achieve a weighted summation of alleles, representing a PRS for ASD risk. 
After evaluating via logistic regression the $R^{2}$ from a model of ASD on ASD-PRS ranging across the discovery thresholds and adjusting for genetic ancestry, we determined that a $p_{\text {discovery }}$ of 0.05 achieved the best fit, and thus used this score in further analyses. The association of the 22q13.33 co-methylated block \% methylation, taken from individual smoothed chr22q block methylation levels obtained using bsseq [94], and diagnosis with PRS was tested by analysis of variance (ANOVA), with replication by linear regression, with PRS as the dependent variable (Additional File 15: Table S14).

\section{Participant genomic insertion characterization and Sanger sequencing}

To validate the 22q13.33 insertion from Illumina WGS data, the expected genomic location of the insertion was queried in a published PacBio long read sequencing dataset [43]. The insertion was identified as located at the CHM1_chr22-49029645-INS-1673 contig [43]. The contig was in a fasta file with accession number GCA_003709635.1 with the correspondence table, it was also named with GenBank ID QPKN01007947.1 in the NCBI database [44]. SAMtools was utilized to isolate the fasta sequence from the contig (85,271 bp in length) and extract the insertion sequence (1673 bp in length) (Additional File 14: Table S13). The QPKN01007947.1 contig mapped to chr22: 49,381,532-49,466,902 (reference genome: hg19) using blat [122] and the insertion was visualized using Miropeats [123].

In addition to characterizing the insertion using PacBio long read sequencing, primer sets were designed to span the insertion location for PCR-based genotyping (Additional File 14: Table S13). A $25 \mu \mathrm{l}$ PCR reaction mixture contained $100 \mathrm{ng}$ genomics DNA, $5 \mu \mathrm{l} 5 \times$ LongAmp Taq reaction buffer (NEB, Ipswich, MA, USA), $1 \mu$ L LongAmp Taq DNA polymerase (NEB, Ipswich, MA, USA), $1 \mu \mathrm{l} 10 \mathrm{mM}$ dNTPs, and $2 \mu \mathrm{l}$ of $10 \mu \mathrm{M}$ forward and reverse primer. The PCR amplifications were performed using following conditions: initial denaturation at $94{ }^{\circ} \mathrm{C}$ for $30 \mathrm{~s} ; 30$ cycles of denaturing at $94{ }^{\circ} \mathrm{C}$ for $30 \mathrm{~s}$, $52{ }^{\circ} \mathrm{C}$ for $30 \mathrm{~s}$, and $65^{\circ} \mathrm{C}$ for 2 min with a final extension at $65^{\circ} \mathrm{C}$ for $10 \mathrm{~min}$. PCR products were subjected to Topoisomerase (TOPO) PCR Cloning Kit (Thermo Fisher Scientific, Waltham, MA, USA) followed by a 1.5\% agarose gel electrophoresis with purification and Sanger sequencing by University of California, Davis DNA Sequencing Facility (Davis, CA, USA), and chromatograms were analyzed using SnapGene (Genewiz, South Plainfield, NJ, USA). PCR product genotype and size were characterized using the Bioanalyzer 2100 (Agilent, Santa Clara, CA, USA). The sequence of the insertion was analyzed for repetitive elements using CENSOR and RepeatMasker [124, 125].

\section{Cell culture, cell-based assays, and transfection}

LUHMES cells (ATCC, Manassas, VA, USA, CRL-2927) were seeded on fibronectincoated plates (Thermo Fisher Scientific, Waltham, MA, USA, CWP001, 354402). Undifferentiated cells were maintained in proliferation medium: Advanced DMEM/F12 (Invitrogen, Carlsbad, CA, USA), supplemented with N2 supplement (Invitrogen, Carlsbad, CA, USA), Penicillin-streptomycin-glutamine (Thermo Fisher Scientific, Waltham, MA, USA), and $40 \mathrm{ng} / \mathrm{ml}$ recombinant bFGF (Invitrogen, Carlsbad, CA, USA). To generate differentiated LUHMES, cells were switched to differentiation media for 5 days. Differentiation media comprised advanced DMEM/F12, supplemented with N2 supplement, Penicillin-streptomycin-glutamine, $1 \mathrm{mM}$ dbcAMP (MilliporeSigma, Burlington, MA, 
USA), $1 \mu \mathrm{g} / \mathrm{ml}$ tetracycline (Neta Scientific, Hainesport, NJ, USA), and $2 \mathrm{ng} / \mathrm{ml}$ recombinant human GDNF (Thermo Fisher Scientific, Waltham, MA, USA). For cell viability and hydrogen peroxide production experiments, differentiated cells were grown in 96well plates for 6 days prior to treatment with CellTiter Blue or ROS-Glo visualization reagent (Promega, Madison, WI, USA). Undifferentiated cells were plated in 96-well plates at the same densities as differentiated neurons and treated identically for cell viability and hydrogen peroxide measurements. For RNA quantification, cells were maintained in 6-well plates. Challenges with hydrogen peroxide (MilliporeSigma, Burlington, MA, USA), cobalt chloride (Thermo Fisher Scientific, Waltham, MA, USA), or mock treatment were carried out after 5 days of differentiation, and cells were treated for 24 $\mathrm{h}$ before analysis.

An overexpression NHIP plasmid, NHIP-eGFP, was synthesized by VectorBuilder (Chicago, IL, USA) with EF- $1 \alpha$ as the promoter for NHIP and CMV as the promoter for eGFP fused with a puromycin resistance gene (Additional File 12: Table S11, Additional File 3: Fig. S18). A control plasmid was cut using XbaI and AbaI restriction endonucleases based on NHIP-eGFP, named NEG-eGFP, with NHIP removed and the rest of plasmid structure maintained (Additional File 12: Table S11, Additional File 3: Fig. S18). The plasmid for the NHIP peptide, NHIP peptide-eGFP, was synthesized by VectorBuilder with EF-1 $\alpha$ as the promoter for the NHIP peptide, and the stop codon removed and fused to the end of the NHIP peptide with eGFP, together with CMV as the promoter for mCherry fused with a puromycin resistance gene (Additional File 12: Table S11, Fig. 2g). All constructs were sequenced with Sanger sequencing by the University of California, Davis, DNA Sequencing Facility (Davis, CA, USA) and analyzed using SnapGene (Genewiz, South Plainfield, NJ, USA) to confirm the expected sequence.

HEK293T cells (ATCC, Manassas, VA, USA, CRL-11268) were grown in DMEM/ F12, GlutaMAX medium (Thermo Fisher Scientific, Waltham, MA, USA) supplemented with MEM non-essential amino acids (Thermo Fisher Scientific, Waltham, MA, USA) and 10\% fetal bovine serum (Invitrogen, Carlsbad, CA, USA) together with Penicillin-streptomycin-glutamine. Low passage HEK293T cells were transfected with plasmids using Lipofectamine 3000 and Opti-MEM (Invitrogen, Carlsbad, CA, USA) according to the manufacturer's instructions. Transfections were performed using HEK293T cell lines for each condition. Transfection medium was replaced $24 \mathrm{~h}$ posttransfection with complete growth media with puromycin at $3 \mu \mathrm{g} / \mathrm{ml}$ for 7 days.

All cells were maintained at $37{ }^{\circ} \mathrm{C}$ containing $95 \% \mathrm{O}_{2}$ and $5 \% \mathrm{CO}_{2}$. Images were taken using an EVOS microscope under the magnification labeled in the images. Cell numbers were measured using the Countess II FL automated cell counter (Thermo Fisher Scientific, Waltham, MA, USA) under the default steps with mixing $10 \mu \mathrm{l}$ of samples with $10 \mu \mathrm{l}$ of trypan blue. CellTiter Blue reagent was used to measure cell viability using luminescence based on manufacturer instructions (Promega, Madison, WI, USA). $\mathrm{H}_{2} \mathrm{O}_{2}$ production representing relative reactive oxygen species (ROS) level was measured with the ROS-Glo $\mathrm{H}_{2} \mathrm{O}_{2}$ assay system using $50 \mathrm{nM}$ with the default settings with level measured by luminometer (Promega, Madison, WI, USA).

HEK293T whole cell lysates were prepared by resuspension in $1 \times$ RIPA buffer and sonication using a Diagenode Bioruptor 300 (Diagenode, Denville, NJ, USA) followed by centrifugation at $21,130 \times g$ at $4{ }^{\circ} \mathrm{C}$ to remove insoluble material and then resolved on 
a 4-15\% SDS-PAGE gel (Biorad, Hercules, CA, USA). The SDS-PAGE gel was rinsed in three changes of water to remove SDS and stained with Imperial protein stain (Thermo Fisher Scientific, Waltham, MA, USA) to visualize proteins. Stained bands between 25 and $37 \mathrm{kDa}$ were carefully excised from the gel, washed in three changes of $50 \mathrm{mM}$ ammonium bicarbonate followed by three washes with acetonitrile, then swollen in $10 \mathrm{mM}$ DTT in acetonitrile and incubated at $56^{\circ} \mathrm{C}$ for $30 \mathrm{~min}$ to reduce disulfide bonds. The gel pieces were next shrunk by incubation in acetonitrile then incubated in $55 \mathrm{mM}$ iodoacetamide (IAA) in $50 \mathrm{mM}$ ammonium bicarbonate prior to washing with $50 \mathrm{mM}$ ammonium bicarbonate, then shrunk with acetonitrile and dried in a speed vac. Gel pieces were suspended in $50 \mathrm{mM}$ ammonium bicarbonate with $0.01 \%$ Protease Max (Promega, Madison, WI, USA) and treated with trypsin (Promega) for $4 \mathrm{~h}$ at $50^{\circ} \mathrm{C}$. The NHIP/GFP fusion protein was detected from the resulting peptides by (LC/MS-MS). MS was performed at University of California, Davis Proteomics Core Facility.

NHIP peptide immunofluorescence staining utilized a custom polyclonal antibody that was produced in Rabbit by GenScript Inc (Piscataway, NJ, USA) to a truncated NHIP peptide MVRGEATARTEEAMC and affinity purified. Flash frozen human cortical tissues were fixed in $4 \%$ formaldehyde in $1 \times$ PBS for $72 \mathrm{~h}$ then dehydrated by immersion in $70 \%$ ethanol for 7 days and embedded in paraffin. Five-micrometer sections were cut from embedded brain tissue and mounted on glass slides then baked for $4 \mathrm{~h}$ at $56^{\circ} \mathrm{C}$. Tissues on slides were washed in four changes of xylene to remove paraffin. Next, slides were washed in two changes of $100 \%$ ethanol which was removed by heating to $50^{\circ} \mathrm{C}$ on a heat block. The slides were then treated with $1 \times$ DAKO antigen retrieval solution (Agilent, Santa Clara, CA, USA) at $95^{\circ} \mathrm{C}$ for $1 \mathrm{~h}$ in a water bath. Slides were washed five times in $1 \times$ PBS with agitation. To reduce endogenous autofluorescence, slides were immersed in $1 \times$ PBS and exposed to LED light for $24 \mathrm{~h}$. Slides were next incubated with $1 \times \mathrm{PBS} / 0.5 \%$ Tween $20 / 3 \% \mathrm{BSA}$ for $1 \mathrm{~h}$ at $37^{\circ} \mathrm{C}$ to block background signals then washed three times in $1 \times \mathrm{PBS} / 0.5 \%$ Tween 20 . Anti-NHIP peptide and control pre-immune antibodies were diluted 1/200 in $1 \times \mathrm{PBS} / 0.5 \%$ Tween 20/3\% BSA and incubated on slides at $37^{\circ} \mathrm{C}$ overnight in a humid chamber before three washes in $1 \times \mathrm{PBS} / 0.5 \%$ Tween. Goat anti-Rabbit Alexa 594 (Thermo Fisher Scientific, Waltham, MA, USA, Catalog \#A32740) was diluted in $1 \times$ PBS/ 0.5\% Tween20/ 3\% BSA with $5 \mu \mathrm{g} / \mathrm{ml}$ DAPI and added to slides for $2 \mathrm{~h}$ at $37^{\circ} \mathrm{C}$ in a humid chamber. Slides were washed five times in $1 \times$ PBS/ $0.5 \%$ Tween 20 with shaking before mounting in $5 \mu \mathrm{g} / \mathrm{ml}$ DAPI in $50 \%$ glycerol and application of glass coverslips.

\section{RNA extraction, CDNA synthesis, and RT-PCR}

Total RNA was isolated from HEK293T cells transiently transfected with NHIP-eGFP or negative control NEG-eGFP using the AllPrep DNA/RNA/Protein mini kit (Qiagen, Hilden, Germany). Human tissue total RNA samples were obtained commercially, including placenta (Life Technology, Carlsbad, CA, USA), testes (TaKaRa Bio, Kusatsu, Shiga, Japan), and fetal brain (Cell Applications, San Diego, CA, USA). RNA was extracted from frozen placenta samples in the Discovery group samples using TRIzol Reagent (Invitrogen, Carlsbad, CA, USA). cDNA was synthesized using the High-Capacity cDNA Reverse Transcription Kit (Thermo Fisher Scientific, Waltham, MA, USA) based on the manufacturer's protocol. TaqMan Gene Expression Assays for LOC105373085 
(renamed as NHIP) (assay ID: Hs01034248_s1), BRD1 (Hs00205849_m1), FAM19A5 (Hs00395354_m1), and GAPDH (assay ID: Hs02786624_g1) were used (Thermo Fisher Scientific, Waltham, MA, USA). The expression of 3 genes of interest and 1 reference genes were examined by real-time TaqMan PCR assay (Thermo Fisher Scientific, Waltham, MA, USA). Expression levels were determined by the probes with optimized primer and probe concentrations. Quantification was accomplished with RT-PCR machine using TaqMan Fast Advanced Master Mix with the default parameters by the manufacturer (Thermo Fisher Scientific, Waltham, MA, USA). Reactions were performed with three biological replicates. Fold changes of transcript levels were measured using the Fluidigm Real-Time PCR Analysis software with fold change of gene expression calculated as the delta delta CT normalized to GAPDH (Fluidigm, San Francisco, CA, USA).

\section{Brain sample acquisition}

Human brain samples were obtained from the NICHD Brain and Tissue Bank for Developmental Disorders at the University of Maryland (Baltimore, MD, USA) (Additional File 16: Table S15). RNA from the frozen human brain was purified using AllPrep DNA/RNA/Protein mini kit (Qiagen, Hilden, Germany).

\section{RNA-seq library preparation and sequencing}

RNA from cells and brain was prepared as RNA-seq libraries using Kapa RNA HyperPrep kits (Roche, Basel, Switzerland) together with the QIAseq FastSelect Human ribodepletion kit (Qiagen, Hilden, Germany). Libraries were assessed for quality and quantified on the Agilent Bioanalyzer 2100, and then pooled for multiplex sequencing with at least 25 million reads with 150 bp paired-end reads on the Illumina NovaSeq 6000 S4 (San Diego, CA, USA) by the DNA Tech Core at University of California, Davis (Davis, CA, USA).

\section{RNA-seq data processing and differential gene expression (DGE)}

Raw fastq files were processed and aligned using STAR [126]. After quality control steps by FASTQC, the count matrixes were generated by featureCounts [127, 128]. Count matrixes were filtered for at least one count in any sample. Size factor estimation and normalization were performed by DESeq2 [129]. DGE was generated compared between overexpressed NHIP and negative control cells using DESeq2 (FDR-corrected $p$ value $<0.05$ ) [129]. DGE for brain was analyzed by using normalized read count for NHIP levels as continuous trait using DESeq2 (FDR-corrected $p$ value $<0.05$ ) [129]. In brain, the normalized read count for NHIP transcripts were adjusted for potential covariates of sex, age, brain region, and PMI using DESeq2 (FDR-corrected $p$ value < 0.05). Gene overlaps between different experiments were tested for significance using Fisher's exact test in the GeneOverlap R package compared to all genes expressed in at least one read in any sample [130].

Gene Ontology terms for DGE were identified using clusterProfiler on Gene Set Enrichment Analysis using gseGO function with 1000 permutations [131]. Normalized enrichment scores (NES) were calculated for enrichment after correcting for multiple testing with FDR. The dotplots illustrate significant GO terms based on GeneRatio, 
calculated from the number of overlapped genes divided by the total number of genes in the gene set [131]. GO terms to be included in the plots were selected based on GeneRatio ranking. The enrichment map was plotted using emapplot function by clustering mutually overlapping gene sets to form functional modules [131]. The ridgeplot was plotted using the ridgeplot $\mathrm{R}$ function to visualize expression distributions of core enriched genes [131]. The cnetplot depicted the linkages of genes and biological concepts as networks [131].

\section{Supplementary Information}

The online version contains supplementary material available at https://doi.org/10.1186/s13059-022-02613-1.

Additional file 1: Table S1. Subject characteristics in relation to outcomes at 36 months.

Additional file 2: Table S2. Subject characteristics by subject.

Additional file 3. This file contains Figures S1-S44.

Additional file 4: Table S3. ASD DMRs identified in discovery group with annotations.

Additional file 5: Table S4. ChromHMM-defined chromatin state enrichment for DMRs.

Additional file 6: Table S5. Transcription factor motif enrichment for DMRs.

Additional file 7: Table S6. Genes in common between DMRs associated genes and SFARI ASD genes.

Additional file 8: Table S7. Gene Ontology terms on ASD DMRs.

Additional file 9: Table S8. 22q13.33 comethylated block replicates with independent studies, and sequencing platforms.

Additional file 10: Table S9. 22q13.33 comethylated block adjusted for nominal covariates (birth weight, nRBC).

Additional file 11: Table S10. Blat results of NHIP in vertebrates.

Additional file 12: Table S11. Plasmid structure for HEK293T cells and NHIP peptide sequence.

Additional file 13: Table S12. NHIP peptide blat research results.

Additional file 14: Table S13. Insertion characteristics in the discovery group and primers,

Additional file 15: Table S14. PRS was tested for association on $22 q 13.33$ comethylated block \% methylation or diagnosis.

Additional file 16: Table S15. Subject characteristics of postmortem brain samples.

Additional file 17: Table S16. Differential gene expression associated with NHIP in brain.

Additional file 18: Table S17. Differential gene expression associated with NHIP in brain, after adjustment for covariates.

Additional file 19: Table S18. Gene ontology analysis on differentially expressed genes (FDR corrected $p$-value $<0.05$ ) associated with NHIP in brain.

Additional file 20: Table S19. Genes in common between DGE in brain and SFARI ASD genes.

Additional file 21: Table S20. Gene ontology analysis on overlapped genes between DGE in brain and SFARI ASD genes

Additional file 22: Table S21. Differential gene expression with overexpressed NHIP in HEK293T cells.

Additional file 23: Table S22. Gene ontology analysis of differentially expressed genes (FDR corrected $p$-value < 0.05) related to overexpressed NHIP treatment in HEK293T cells.

Additional file 24: Table S23. KEGG pathway enrichment of differentially expressed genes (FDR corrected $p$ value $<0.05$ ) with overexpressed NHIP treatment in HEK293T cells.

Additional file 25: Table S24. Genes in common between DGE in brain and DGE in cells.

Additional file 26: Table S25. Gene ontology analysis on the overlapped genes between DGE in brain and DGE in cells.

Additional file 27: Table S26. Gene ontology in common between significant gene ontology terms for brain DGE and significant terms for cell DGE.

Additional file 28: Table S27. Genes in common between DGE in brain and SFARI ASD genes.

Additional file 29: Table S28. Gene ontology analysis on the overlapped genes between DGE in cells and SFARI ASD genes.

Additional file 30: Table S29. Genes in common among DGE in brain, DGE in cells, and SFARI ASD genes. Additional file 31: Table S30. Gene ontology analysis on the overlapped genes among DGE in brain, DGE in cells, and SFARI ASD genes.

Additional file 32. Review history. 


\section{Acknowledgements}

We would like to thank the MARBLES and EARLI study participants.

\section{Review history}

The review history is available as Additional file 32 .

\section{Peer review information}

Barbara Cheifet was the primary editor of this article and managed its editorial process and peer review in collaboration with the rest of the editorial team.

\section{Authors' contributions}

YZ contributed to sample preparation, bioinformatics analysis, data visualization, interpretation, and writing of the manuscript. JAG designed cell culture experiments, performed cell experiments, and provided substantial assistance with the project. BIL contributed extensively to WGBS data analysis, together with substantial bioinformatics assistance. CEM performed WGS data collection and provided critical advice for bioinformatics data analysis. JSM performed experiments. DCS, MYD, and KSB contributed to genomics analysis. KMB and JD performed data analysis. JMJ, RM, and OJG contributed experiments. LAW performed data analysis. CKW, SO, JD, LPG, HEV, JIF, MDF, IH, and RJS contributed to the study design. DHY provided experiments and critical advice. JML and RJS conceived of the study. JML contributed substantially on study design, data interpretation, and manuscript for this project. All authors read and approved the final manuscript.

\section{Funding}

This work was funded in part by NIH R01 ES029213, R01 ES025574, R01 ES029213, P01 ES011269, R01 ES020392; Environmental Protection Agency (83543201); Intellectual and Developmental Disability Research Centers (P50 HD103526); UC Davis Environmental Health Sciences Center (P30 ES023513); Environmental Influences on Child Health Outcomes (ECHO) Consortium (UH3 ES023365); Canadian Institutes of Health Research (CIHR) post- doctoral fellowship (MFE-146824 to BIL); CIHR Banting postdoctoral fellowship (BPF-162684 to BIL).

\section{Availability of data and materials}

Datasets supporting the conclusions are available in the Gene Expression Omnibus repository (GEO) [132] at accession number (GSE178206) [133]. Code and scripts for this study are available on GitHub [134]. The gene abbreviation NHIP for "neuronal hypoxia inducible, placental associated" for LOC105373085 was approved by the HUGO Gene Nomenclature Committee.

\section{Declarations}

\section{Ethics approval and consent to participate}

This study was performed in accordance with the ethical principles for medical research involving human subjects in the Declaration of Helsinki. The University of California, Davis Institutional Review Board and the State of California Committee for the Protection of Human Subjects approved this study and the MARBLES Study protocols (IRB\# 225645). Human Subjects Institutional Review Boards at each of the four sites in the EARLI Study approved this study and the EARLI Study protocols (IRB\# 214753). Neither data nor specimens were collected until written informed consent to participate was obtained from the parents.

\section{Competing interests}

The authors declare that they have no competing interests.

\section{Author details}

'Department of Medical Microbiology and Immunology, University of California, Davis, CA, USA. ${ }^{2}$ Perinatal Origins of Disparities Center, University of California, Davis, CA, USA. ${ }^{3}$ Genome Center, University of California, Davis, CA, USA. ${ }^{4}$ MIND Institute, School of Medicine, University of California, Davis, CA, USA. ${ }^{5}$ Department of Biochemistry and Molecular Medicine, School of Medicine, University of California, Davis, CA, USA. ${ }^{6}$ Department of Public Health Sciences, University of California, Davis, CA, USA. 'Department of Epidemiology, School of Public Health, University of Michigan, Ann Arbor, MI, USA. ${ }^{8}$ Department of Obstetrics and Gynecology, University of California, Davis, CA, USA. ${ }^{9}$ Department of Psychiatry and Behavioral Sciences, Davis, CA, USA. ${ }^{10}$ Department of Mental Health, Bloomberg School of Public Health, Johns Hopkins University, Baltimore, MD, USA. ${ }^{11}$ Wendy Klag Center for Autism and Developmental Disabilities, Bloomberg School of Public Health, Johns Hopkins University, Baltimore, MD, USA.

Received: 7 September 2021 Accepted: 16 January 2022

Published online: 16 February 2022

\section{References}

1. Maenner MJ, Shaw KA, Baio J, Washington A, Patrick M, DiRienzo M, et al. Prevalence of autism spectrum disorder among children aged 8 years-autism and developmental disabilities monitoring network, 11 Sites, United States, 2016. MMWR Surveill Summ. 2020;69:1-12 Centers for Disease Control and Prevention (CDC). Available from: https://www.cdc. $\mathrm{gov} / \mathrm{mmwr} / \mathrm{volumes} / 69 / \mathrm{ss} / \mathrm{ss} 6904 \mathrm{a} 1 . \mathrm{htm}$.

2. Bourgeron T. From the genetic architecture to synaptic plasticity in autism spectrum disorder. Nat Rev Neurosci. 2015; 16(9):551-63. https://doi.org/10.1038/nrn3992.

3. Hallmayer J, Cleveland S, Torres A, Phillips J, Cohen B, Torigoe T, et al. Genetic heritability and shared environmental factors among twin pairs with autism. Arch Gen Psychiatry. 2011;68(11):1095-102. https://doi.org/10.1001/a rchgenpsychiatry.2011.76. 
4. Mazina V, Gerdts J, Trinh S, Ankenman K, Ward T, Dennis MY, et al. Epigenetics of autism-related impairment: copy number variation and maternal infection. J Dev Behav Pediatr. 2015;36(2):61-7. https://doi.org/10.1097/DBP. 0000000000000126.

5. Gaugler T, Klei L, Sanders SJ, Bodea CA, Goldberg AP, Lee AB, et al. Most genetic risk for autism resides with common variation. Nat Genet. 2014;46(8):881-5. https://doi.org/10.1038/ng.3039.

6. Iossifov I, O'Roak BJ, Sanders SJ, Ronemus M, Krumm N, Levy D, et al. The contribution of de novo coding mutations to autism spectrum disorder. Nature. 2014;515:216-21 Available from: http://www.nature.com/articles/nature13908.

7. Sanders SJ, He X, Willsey AJ, Ercan-Sencicek AG, Samocha KE, Cicek AE, et al. Insights into autism spectrum disorder genomic architecture and biology from 71 risk loci. Neuron. 2015;87:1215-33 Available from: http://www.ncbi.nlm.nih. gov/pubmed/26402605.

8. Grove J, Ripke S, Als TD, Mattheisen M, Walters RK, Won H, et al. Identification of common genetic risk variants for autism spectrum disorder. Nat Genet. 2019;51(3):431-44. https://doi.org/10.1038/s41588-019-0344-8.

9. Clarke TK, Lupton MK, Fernandez-Pujals AM, Starr J, Davies G, Cox S, et al. Common polygenic risk for autism spectrum disorder (ASD) is associated with cognitive ability in the general population. Mol Psychiatry. 2016;21(3):419-25. Available from: https://pubmed.ncbi.nlm.nih.gov/25754080/. https://doi.org/10.1038/mp.2015.12.

10. Satterstrom FK, Kosmicki JA, Wang J, Breen MS, De Rubeis S, An JY, et al. Large-scale exome sequencing study implicates both developmental and functional changes in the neurobiology of autism. Cell. 2020;180:568-84.e23 Available from: https://doi.org/10.1016/j.cell.2019.12.036.

11. Ripke S, Neale BM, Corvin A, Walters JT, Farh KH, Holmans PA, Lee P, Bulik-Sullivan B, Collier DA, Huang H, Pers TH. Biological insights from 108 schizophrenia-associated genetic loci. Nature. 2014;511(7510):421. Available from: https:// www.nature.com/articles/nature13595.

12. Ursini G, Punzi G, Chen Q, Marenco S, Robinson JF, Porcelli A, et al. Convergence of placenta biology and genetic risk for schizophrenia. Nat Med. 2018;24(6):792-801. Available from: http://www.nature.com/articles/s41591-018-0021-y. https://doi.org/10.1038/s41591-018-0021-y.

13. Schroeder DI, Jayashankar K, Douglas KC, Thirkill TL, York D, Dickinson PJ, et al. Early developmental and evolutionary origins of gene body DNA methylation patterns in mammalian placentas. PLoS Genet. 2015;11(8):1-20. https://doi.org/1 0.1371/journal.pgen.1005442.

14. Schroeder DI, Lott P, Korf I, LaSalle JM. Large-scale methylation domains mark a functional subset of neuronally expressed genes. Genome Res. 2011;21:1583-91 Available from: http://www.ncbi.nlm.nih.gov/pubmed/21784875.

15. Schroeder DI, Blair JD, Lott P, Yu HOK, Hong D, Crary F, et al. The human placenta methylome. Proc Natl Acad Sci U S A. 2013;110:6037-42 Available from: http://www.pubmedcentral.nih.gov/articlerender.fcgi?artid=3625261\&tool= pmcentrez\&rendertype=abstract.

16. Lorincz MC, Schübeler D. Evidence for converging DNA methylation pathways in placenta and cancer. Dev Cell. 2017; 43(3):257-8.

17. Zhao H, Wong RJ, Stevenson DK. The impact of hypoxia in early pregnancy on placental cells. Int J Mol Sci. 2021;22(18): 9675. https://doi.org/10.3390/ijms22189675.

18. Vieira HLA, Alves PM, Vercelli A. Modulation of neuronal stem cell differentiation by hypoxia and reactive oxygen species. Prog Neurobiol. 2011;93(3):444-55. https://doi.org/10.1016/j.pneurobio.2011.01.007.

19. Rock KD, Patisaul HB. Environmental mechanisms of neurodevelopmental toxicity. Curr Environ Health Rep. 2018;5(1): 145-57. https://doi.org/10.1007/s40572-018-0185-0.

20. Schroeder DI, Schmidt RJ, Crary-Dooley FK, Walker CK, Ozonoff S, Tancredi DJ, et al. Placental methylome analysis from a prospective autism study. Mol Autism. 2016;7:51 Available from: http://molecularautism.biomedcentral.com/articles/10.11 86/s13229-016-0114-8\%5Cn, http://www.ncbi.nlm.nih.gov/pubmed/28018572.

21. Zhu Y, Mordaunt CE, Yasui DH, Marathe R, Coulson RL, Dunaway KW, et al. Placental DNA methylation levels at CYP2E1 and IRS2 are associated with child outcome in a prospective autism study. Hum Mol Genet. 2019;28:2659-74. Available from: https://academic.oup.com/hmg/article/28/16/2659/5476403. https://doi.org/10.1093/hmg/ddz084.

22. Santos HP, Bhattacharya A, Joseph RM, Smeester L, Kuban KCK, Marsit CJ, et al. Evidence for the placenta-brain axis: multi-omic kernel aggregation predicts intellectual and social impairment in children born extremely preterm. Mol Autism. 2020;11:97 Available from: https://doi.org/10.1186/s13229-020-00402-w.

23. Corley MJ, Vargas-Maya N, Pang APS, Lum-Jones A, Li D, Khadka V, et al. Epigenetic delay in the neurodevelopmental trajectory of DNA methylation states in autism spectrum disorders. Front Genet. 2019;10:907 Available from: https:// www.frontiersin.org/article/10.3389/fgene.2019.00907/full.

24. Gunasekara CJ, Scott CA, Laritsky E, Baker MS, MacKay H, Duryea JD, et al. A genomic atlas of systemic interindividual epigenetic variation in humans. Genome Biol. 2019;20(1):105. https://doi.org/10.1186/s13059-019-1708-1.

25. Kessler NJ, Waterland RA, Prentice AM, Silver MJ. Establishment of environmentally sensitive DNA methylation states in the very early human embryo. Sci Adv. 2018;4:eaat2624.

26. Hollox EJ, Huffmeier U, Zeeuwen PLM, Palla R, Lascorz J, Rodijk-Olthuis D, et al. Psoriasis is associated with increased $\beta$ defensin genomic copy number. Nat Genet. 2008;40(1):23-5. https://doi.org/10.1038/ng.2007.48.

27. Stefansson H, Rujescu D, Cichon S, Pietiläinen OPH, Ingason A, Steinberg S, et al. Large recurrent microdeletions associated with schizophrenia. Nature. 2008;455(7210):232-6. https://doi.org/10.1038/nature07229.

28. Sekar A, Bialas AR, De Rivera H, Davis A, Hammond TR, Kamitaki N, et al. Schizophrenia risk from complex variation of complement component 4. Nature. 2016;530(7589):177-83. https://doi.org/10.1038/nature16549.

29. Gokcumen $\mathrm{O}$, Babb PL, Iskow RC, Zhu Q, Shi X, Mills RE, et al. Refinement of primate copy number variation hotspots identifies candidate genomic regions evolving under positive selection. Genome Biol. 2011;12(5):R52. https://doi.org/1 $0.1186 / g b-2011-12-5-r 52$.

30. Lin YL, Gokcumen O. Fine-scale characterization of genomic structural variation in the human genome reveals adaptive and biomedically relevant hotspots. Genome Biol Evol. 2019;11(4):1136-51. https://doi.org/10.1093/ gbe/evz058.

31. Girirajan S, Brkanac Z, Coe BP, Baker C, Vives L, Vu TH, et al. Relative burden of large CNVs on a range of neurodevelopmental phenotypes. PLoS Genet. 2011;7(11):e1002334. https://doi.org/10.1371/journal.pgen.1002334. 
32. Pinto D, Pagnamenta AT, Klei L, Anney R, Merico D, Regan R, et al. Functional impact of global rare copy number variation in autism spectrum disorders. Nature. 2010;466(7304):368-72. https://doi.org/10.1038/nature09146.

33. Girirajan S, Dennis MY, Baker C, Malig M, Coe BP, Campbell CD, et al. Refinement and discovery of new hotspots of copy-number variation associated with autism spectrum disorder. Am J Hum Genet. 2013;92(2):221-37. https://doi.org/1 0.1016/j.ajhg.2012.12.016.

34. Turner TN, Hormozdiari F, Duyzend MH, McClymont SA, Hook PW, lossifov I, et al. Genome sequencing of autismaffected families reveals disruption of putative noncoding regulatory DNA. Am J Hum Genet. 2016;98(1):58-74. https:// doi.org/10.1016/j.ajhg.2015.11.023.

35. Ernst J, Kellis M. Chromatin-state discovery and genome annotation with ChromHMM. Nat Protoc. 2017;12(12):2478-92. Available from: http://www.nature.com/doifinder/10.1038/nprot.2017.124.

36. Abrahams BS, Arking DE, Campbell DB, Mefford HC, Morrow EM, Weiss LA, et al. SFARI Gene 2.0: a community-driven knowledgebase for the autism spectrum disorders (ASDs). Mol Autism. 2013;4:36 Available from: http://molecularautism. biomedcentral.com/articles/10.1186/2040-2392-4-36.

37. Sloan CA, Chan ET, Davidson JM, Malladi VS, Strattan JS, Hitz BC, et al. ENCODE data at the ENCODE portal. Nucleic Acids Res. 2016;44:D726-32 Available from: http://www.ncbi.nlm.nih.gov/pubmed/26527727.

38. Ota T, Suzuki Y, Nishikawa T, Otsuki T, Sugiyama T, Irie R, et al. Complete sequencing and characterization of 21,243 fulllength human cDNAs. Nat Genet. 2004;36(1):40-5. https://doi.org/10.1038/ng1285.

39. Lonsdale J, Thomas J, Salvatore M, Phillips R, Lo E, Shad S, et al. The Genotype-Tissue Expression (GTEx) project. Nat Genet. 2013:45:580-5 Available from: https://www.nature.com/articles/ng.2653.

40. Ortega JA, Sirois CL, Memi F, Glidden N, Zecevic N. Oxygen levels regulate the development of human cortical radial glia cells. Cereb Cortex. 2017;27:3736-51.

41. Hayashi M, Sakata M, Takeda T, Tahara M, Yamamoto T, Minekawa R, et al. Hypoxia up-regulates hypoxia-inducible factor-1a expression through RhoA activation in trophoblast cells. J Clin Endocrinol Metab. 2005;90(3):1712-9. Available from: https://pubmed.ncbi.nlm.nih.gov/15598682/. https://doi.org/10.1210/jc.2004-1547.

42. Simon MC, Keith B. The role of oxygen availability in embryonic development and stem cell function. Nat Rev Mol Cell Biol. 2008:9(4):285-96. https://doi.org/10.1038/nrm2354.

43. Audano PA, Sulovari A, Graves-Lindsay TA, Cantsilieris S, Sorensen M, Welch AME, et al. Characterizing the major structural variant alleles of the human genome. Cell. 2019;176:663 75.e19.

44. Homo sapiens isolate CHM1 chromosome 22 22-49000000:0, whole genome SH - nucleotide - NCBI [Internet]. National Center for Biotechnology Information. U.S. National Library of Medicine; [cited 2021 Sep 7]. Available from: https:/www. ncbi.nlm.nih.gov/nuccore/QPKN01007947.

45. Collins RL, Brand H, Karczewski KJ, Zhao X, Alföldi J, Francioli LC, et al. A structural variation reference for medical and population genetics. Nature. 2020;581(7809):444-51. https://doi.org/10.1038/s41586-020-2287-8.

46. Collins RL, Brand H, Karczewski K, Zhao X, Alföldi J, Francioli LC, et al. INS_22_115103. Mutat. constraint Spectr. quantified from Var. 141,456 humans. 2020. 434-43. Available from: https://gnomad.broadinstitute.org/variant/INS_22_11 5103? dataset=gnomad_SV_r2_1

47. Schmidt RJ, losif A-M, Guerrero Angel E, Ozonoff S. Association of maternal prenatal vitamin use with risk for autism spectrum disorder recurrence in young siblings. JAMA Psychiatry. 2019;76:391 Available from: http://archpsyc.jama network.com/article.aspx?doi=10.1001/jamapsychiatry.2018.3901.

48. Braun JM, Froehlich T, Kalkbrenner A, Pfeiffer CM, Fazili Z, Yolton K, Lanphear BP. Brief report: are autistic-behaviors in children related to prenatal vitamin use and maternal whole blood folate concentrations? J Autism Dev Disord. 2014; 44(10):2602-7. Available from: https://link.springer.com/article/10.1007\%2Fs10803-014-2114-x.

49. Schmidt RJ, Hansen RL, Hartiala J, Allayee H, Schmidt LC, Tancredi DJ, et al. Prenatal vitamins, one-carbon metabolism gene variants, and risk for autism. Epidemiology. 2011;22:476-85 Available from: http://www.ncbi.nlm.nih.gov/ pubmed/21610500.

50. Brieger KK, Bakulski KM, Pearce CL, Baylin A, Dou JF, Feinberg Jl, et al. The association of prenatal vitamins and folic acid supplement intake with odds of autism spectrum disorder in a high-risk sibling cohort, the Early Autism Risk Longitudinal Investigation (EARLI). J Autism Dev Disord. 2021. https://doi.org/10.1007/s10803-021-05110-9.

51. Kaiser VB, Semple CA. Chromatin loop anchors are associated with genome instability in cancer and recombination hotspots in the germline. Genome Biol. 2018;19:101 Available from: https:/pubmed.ncbi.nlm.nih.gov/30060743/.

52. Boix CA, James BT, Park YP, Meuleman W, Kellis M. Regulatory genomic circuitry of human disease loci by integrative epigenomics. Nature. 2021;590:300-7 Available from: https://doi.org/10.1038/s41586-020-03145-z.

53. Schmitt AD, Hu M, Jung I, Xu Z, Qiu Y, Tan CL, et al. A compendium of chromatin contact maps reveals spatially active regions in the human genome. Cell Rep. 2016;17(8):2042-59. https://doi.org/10.1016/j.celrep.2016.10.061.

54. Bernier R, Golzio C, Xiong B, Stessman HA, Coe BP, Penn O, et al. Disruptive CHD8 mutations define a subtype of autism early in development. Cell. 2014;158(2):263-76. https://doi.org/10.1016/j.cell.2014.06.017.

55. Kanehisa M, Goto S. KEGG: Kyoto Encyclopedia of Genes and Genomes. Nucleic Acids Res. 2000;28:27 Available from: /pmc/articles/PMC102409/.

56. Smallwood SA, Kelsey G. De novo DNA methylation: a germ cell perspective. Trends Genet. 2012;28(1):33-42. https://doi. org/10.1016/j.tig.2011.09.004.

57. Moessner R, Marshall CR, Sutcliffe JS, Skaug J, Pinto D, Vincent J, et al. Contribution of SHANK3 mutations to autism spectrum disorder. Am J Hum Genet. 2007;81(6):1289-97. https://doi.org/10.1086/522590.

58. Johannessen M, Haugen IB, Bakken TL, Braaten $\varnothing$. A 22q13.33 duplication harbouring the SHANK3 gene: Does it cause neuropsychiatric disorders? BMJ Case Rep. 2019;12:e228258.

59. Han K, Holder JL, Schaaf CP, Lu H, Chen H, Kang H, et al. SHANK3 overexpression causes manic-like behaviour with unique pharmacogenetic properties. Nature. 2013;503(7474):72-7. https://doi.org/10.1038/nature12630.

60. Marshall CR, Noor A, Vincent JB, Lionel AC, Feuk L, Skaug J, et al. Structural variation of chromosomes in autism spectrum disorder. Am J Hum Genet. 2008;82(2):477-88. https://doi.org/10.1016/.j.ajhg.2007.12.009.

61. Pfaender S, Sauer AK, Hagmeyer S, Mangus K, Linta L, Liebau S, et al. Zinc deficiency and low enterocyte zinc transporter expression in human patients with autism related mutations in SHANK3. Sci Rep. 2017;7(1):1-15. https://doi. org/10.1038/srep45190. 
62. Dennis MY, Eichler EE. Human adaptation and evolution by segmental duplication. Curr Opin Genet Dev. 2016;41:44-52. https://doi.org/10.1016/j.gde.2016.08.001.

63. Carter AM. Placental oxygen consumption. Part I: In vivo studies - a review. Placenta. 2000;21:S31-7. https://doi.org/10.1 053/plac.1999.0513.

64. Rosenfeld CS. The placenta-brain-axis. J Neurosci Res. 2021;99(1):271-83. https://doi.org/10.1002/jnr.24603.

65. Zamudio S, Wu Y, letta F, Rolfo A, Cross A, Wheeler T, et al. Human placental hypoxia-inducible factor-1a expression correlates with clinical outcomes in chronic hypoxia in vivo. Am J Pathol. 2007;170(6):2171-9. https://doi.org/10.2353/a jpath.2007.061185.

66. Semenza GL. Regulation of oxygen homeostasis by hypoxia-Inducible factor 1. Physiology. 2009;24(2):97-106. https:// doi.org/10.1152/physiol.00045.2008.

67. Genbacev O, Zhou Y, Ludlow JW, Fisher SJ. Regulation of human placental development by oxygen tension. Science. 1997;277:1669-72.

68. Sun L, Macgowan CK, Sled JG, Yoo SJ, Manlhiot C, Porayette P, et al. Reduced fetal cerebral oxygen consumption is associated with smaller brain size in fetuses with congenital heart disease. Circulation. 2015;131(15):1313-23. https://doi. org/10.1161/CIRCULATIONAHA.114.013051.

69. Turner JM, Mitchell MD, Kumar SS. The physiology of intrapartum fetal compromise at term. Am J Obstet Gynecol. 2020; 222(1):17-26. https://doi.org/10.1016/.j.ajog.2019.07.032.

70. Fajersztajn L, Veras MM. Hypoxia: from placental development to fetal programming. Birth Defects Res. 2017;109(17): 1377-85. https://doi.org/10.1002/bdr2.1142.

71. Raz R, Roberts AL, Lyall K, Hart JE, Just AC, Laden F, et al. Autism spectrum disorder and particulate matter air pollution before, during, and after pregnancy: a nested case-control analysis within the Nurses' Health Study II Cohort. Environ Health Perspect. 2015;123(3):264-70. Available from: https://ehp.niehs.nih.gov/doi/10.1289/ehp.1408133.

72. Roberts EM, English PB, Grether JK, Windham GC, Somberg L, Wolff C. Maternal residence near agricultural pesticide applications and autism spectrum disorders among children in the California Central Valley. Environ Health Perspect. 2007;115(10):1482-9. https://doi.org/10.1289/ehp.10168.

73. Fagiolini M, Jensen CL, Champagne FA. Epigenetic influences on brain development and plasticity. Curr Opin Neurobiol. 2009;19(2):207-12. https://doi.org/10.1016/j.conb.2009.05.009

74. Ladd-Acosta C, Hansen KD, Briem E, Fallin MD, Kaufmann WE, Feinberg AP. Common DNA methylation alterations in multiple brain regions in autism. Mol Psychiatry. 2014;19(8):862-71. Available from: https://www.nature.com/articles/mp2 013114.

75. Dunaway KW, Islam MS, Coulson RL, Lopez SJ, Vogel Ciernia A, Chu RG, et al. Cumulative impact of polychlorinated biphenyl and large chromosomal duplications on DNA methylation, chromatin, and expression of autism candidate genes. Cell Rep. 2016;17(11):3035-48. https://doi.org/10.1016/j.celrep.2016.11.058.

76. Nardone S, Sams DS, Zito A, Reuveni E, Elliott E. Dysregulation of cortical neuron DNA methylation profile in autism spectrum disorder. Cereb Cortex. 2017;27(12):5739-54. Available from: https://academic.oup.com/cercor/article/27/12/573 9/4259743.

77. Hertz-Picciotto I, Schmidt RJ, Walker CK, Bennett DH, Oliver M, Shedd-Wise KM, et al. A prospective study of environmental exposures and early biomarkers in autism spectrum disorder: design, protocols, and preliminary data from the MARBLES study. Environ Health Perspect. 2018;126(11):117004. Available from: https://ehp.niehs.nih.gov/doi/1 0.1289/EHP535.

78. Mordaunt CE, Jianu JM, Laufer BI, Zhu Y, Hwang H, Dunaway KW, et al. Cord blood DNA methylome in newborns later diagnosed with autism spectrum disorder reflects early dysregulation of neurodevelopmental and X-linked genes. Genome Med. 2020;12:88 Available from: https://genomemedicine.biomedcentral.com/articles/10.1186/s13073-02000785-8.

79. Zhu Y, Mordaunt CE, Durbin-Johnson BP, Caudill MA, Malysheva OV, Miller JW, et al. Expression changes in epigenetic gene pathways associated with one-carbon nutritional metabolites in maternal blood from pregnancies resulting in autism and non-typical neurodevelopment. Autism Res. 2020;14(1):11-28. Available from: https://onlinelibrary.wiley.com/ doi/full/10.1002/aur.2428.

80. Newschaffer CJ, Croen LA, Fallin MD, Hertz-Picciotto I, Nguyen DV, Lee NL, et al. Infant siblings and the investigation of autism risk factors. J Neurodev Disord. 2012;4:7 Available from: http://www.jneurodevdisorders.com/content/4/1/7.

81. Ladd-Acosta C, Andrews SV, Bakulski KM, Feinberg Jl, Tryggvadottir R, Yao R, et al. Placenta DNA methylation at ZNF300 is associated with fetal sex and placental morphology. bioRxiv. 2021; Available from: https://doi.org/10.1101/2021.03. 05.433992.

82. Lord C, Risi S, Lambrecht L, Cook EH, Leventhal BL, DiLavore PC, et al. Autism diagnostic observation schedule (ADOS). J Autism Dev Disord. 2000;30(3):205-23. https://doi.org/10.1023/A:1005592401947.

83. Rutter M, LeCouteur A, Lord C. Autism Diagnostic Interview - Revised (ADI-R). Statew Agric L Use Baseline. 2015;2015:1.

84. Mullen E. Mullen scales of early learning. 1995. Available from: http://www.v-psyche.com/doc/special-cases/MullenSca lesofEarlyLearning.docx

85. Chawarska K, Shic F, Macari S, Campbell DJ, Brian J, Landa R, et al. 18-month predictors of later outcomes in younger siblings of children with autism spectrum disorder: a baby siblings research consortium study. J Am Acad Child Adolesc Psychiatry. 2014;53:1317-27.e1 Available from: http://www.ncbi.nlm.nih.gov/pubmed/25457930.

86. Ozonoff S, Young GS, Belding A, Hill M, Hill A, Hutman T, et al. The broader autism phenotype in infancy: when does it emerge? J Am Acad Child Adolesc Psychiatry. 2014;53(4):398-407. https://doi.org/10.1016/j.jaac.2013.12.020.

87. Laufer Bl, Hwang H, Jianu JM, Mordaunt CE, Korf IF, Hertz-Picciotto I, et al. Low-pass whole genome bisulfite sequencing of neonatal dried blood spots identifies a role for RUNX1 in down syndrome DNA methylation profiles. Hum Mol Genet. 2020;29(21):3465-76. https://doi.org/10.1093/hmg/ddaa218.

88. Krueger F, Andrews SR. Bismark: a flexible aligner and methylation caller for Bisulfite-Seq applications. Bioinformatics. 2011;27(11):1571-2. https://doi.org/10.1093/bioinformatics/btr167.

89. Coulson RL, Yasui DH, Dunaway KW, Laufer BI, Vogel Ciernia A, Zhu Y, et al. Snord116-dependent diurnal rhythm of DNA methylation in mouse cortex. Nat Commun. 2018;9:1616. https://doi.org/10.1038/s41467-018-03676-0. 
90. Krueger F. Trim Galore!: A wrapper tool around Cutadapt and FastQC to consistently apply quality and adapter trimming to FastQ files. Babraham Inst. 2015. Available from: https://www.bioinformatics.babraham.ac.uk/projects/trim_ galore/.

91. Langmead B, Salzberg SL. Fast gapped-read alignment with Bowtie 2. Nat Methods. 2012;9(4):357-9. https://doi.org/10.1 038/nmeth.1923.

92. Li H, Handsaker B, Wysoker A, Fennell T, Ruan J, Homer N, et al. The Sequence Alignment/Map format and SAMtools. Bioinformatics. 2009;25(16):2078-9. Available from: https://pubmed.ncbi.nlm.nih.gov/19505943/. https://doi.org/10.1093/ bioinformatics/btp352.

93. Ewels $P$, Magnusson M, Lundin S, Käller M. MultiQC: summarize analysis results for multiple tools and samples in a single report. Bioinformatics. 2016;32(19):3047-8. https://doi.org/10.1093/bioinformatics/btw354.

94. Hansen KD, Langmead B, Irizarry RA. BSmooth: from whole genome bisulfite sequencing reads to differentially methylated regions. Genome Biol. 2012;13(10):1-10. https://doi.org/10.1186/gb-2012-13-10-r83.

95. Mordaunt CE, Kieffer DA, Shibata NM, Członkowska A, Litwin T, Weiss K-H, et al. Epigenomic signatures in liver and blood of Wilson disease patients include hypermethylation of liver-specific enhancers. Epigenetics Chromatin. 2019;12: 10 Available from: https://epigeneticsandchromatin.biomedcentral.com/articles/10.1186/s13072-019-0255-z.

96. Vu VQ. ggbiplot: A ggplot2 based biplot. R package version 0.55. 2011.

97. Aryee MJ, Jaffe AE, Corrada-Bravo H, Ladd-Acosta C, Feinberg AP, Hansen KD, et al. Minfi: a flexible and comprehensive Bioconductor package for the analysis of Infinium DNA methylation microarrays. Bioinformatics. 2014;30(10):1363-9. Available from: https://academic.oup.com/bioinformatics/article-lookup/doi/10.1093/bioinformatics/btu049.

98. Triche TJ, Weisenberger DJ, Van Den Berg D, Laird PW, Siegmund KD. Low-level processing of illumina infinium DNA methylation beadarrays. Nucleic Acids Res. 2013;41(7):e90. https://doi.org/10.1093/nar/gkt090.

99. Yuan V, Price EM, Del Gobbo G, Mostafavi S, Cox B, Binder AM, et al. Accurate ethnicity prediction from placental DNA methylation data. Epigenetics Chromatin. 2019;12(1):1-14. https://doi.org/10.1186/s13072-019-0296-3.

100. Laufer B. GitHub - ben-laufer/DMRichR: an executable and package for the statistical analysis and visualization of differentially methylated regions (DMRs) from CpG count matrices (Bismark cytosine reports) [Internet]. GitHub. [cited 2021 Sep 7]. Available from: https://github.com/ben-laufer/DMRichR.

101. Korthauer K, Chakraborty S, Benjamini Y, Irizarry RA. Detection and accurate false discovery rate control of differentially methylated regions from whole genome bisulfite sequencing. Biostatistics. 2019;20:367-83. Available from: https://aca demic.oup.com/biostatistics/article/20/3/367/4899074. https://doi.org/10.1093/biostatistics/kxy007.

102. McLean CY, Bristor D, Hiller M, Clarke SL, Schaar BT, Lowe CB, et al. GREAT improves functional interpretation of cisregulatory regions. Nat Biotechnol. 2010;28:495-501 Available from: http://www.ncbi.nlm.nih.gov/pubmed/20436461.

103. Sheffield NC, Bock C. LOLA: enrichment analysis for genomic region sets and regulatory elements in $\mathrm{R}$ and Bioconductor. Bioinformatics. 2016;32:587-9 Available from: http://www.ncbi.nlm.nih.gov/pubmed/26508757.

104. Kundaje A, Meuleman W, Ernst J, Bilenky M, Yen A, Heravi-Moussavi A, et al. Integrative analysis of 111 reference human epigenomes. Nature. 2015;518:317-30 Available from: http://www.nature.com/articles/nature14248.

105. Cavalcante RG, Sartor MA. Annotatr: Genomic regions in context. Bioinformatics. 2017;33(15):2381-3. https://doi.org/10.1 093/bioinformatics/btx183.

106. ENCODE Project Consortium TEP. An integrated encyclopedia of DNA elements in the human genome. Nature. 2012; 489:57-74 Available from: http://www.ncbi.nlm.nih.gov/pubmed/22955616.

107. Heinz S, Benner C, Spann N, Bertolino E, Lin YC, Laslo P, et al. Simple combinations of lineage-determining transcription factors prime cis-regulatory elements required for macrophage and B cell identities. Mol Cell. 2010;38(4):576-89. https:// doi.org/10.1016/j.molcel.2010.05.004.

108. Li H, Durbin R. Fast and accurate short read alignment with Burrows-Wheeler transform. Bioinformatics. 2009;24(14): 1754-60. https://doi.org/10.1093/bioinformatics/btp324.

109. Broad Institute. Picard tools - by broad institute. Github. 2009.

110. McKenna A, Hanna M, Banks E, Sivachenko A, Cibulskis K, Kernytsky A, et al. The genome analysis toolkit: a MapReduce framework for analyzing next-generation DNA sequencing data. Genome Res. 2010;20(9):1297-303. Available from: http://genome.cshlp.org/cgi/doi/10.1101/gr.107524.110.

111. Wang K, Li M, Hakonarson H. ANNOVAR: functional annotation of genetic variants from high-throughput sequencing data. Nucleic Acids Res. 2010;38(16):e164. Available from: https://academic.oup.com/nar/article-lookup/doi/10.1093/nar/ gkq603.

112. Boeva V, Popova T, Bleakley K, Chiche P, Cappo J, Schleiermacher G, et al. Control-FREEC: A tool for assessing copy number and allelic content using next-generation sequencing data. Bioinformatics. 2012;28(3):423-5. https://doi.org/10.1 093/bioinformatics/btr670.

113. Wang J, Mullighan CG, Easton J, Roberts S, Heatley SL, Ma J, et al. CREST maps somatic structural variation in cancer genomes with base-pair resolution. Nat Methods. 2011;8(8):652-4. https://doi.org/10.1038/nmeth.1628.

114. Rausch T, Zichner T, Schlattl A, Stütz AM, Benes V, Korbel JO. DELLY: Structural variant discovery by integrated pairedend and split-read analysis. Bioinformatics. 2012;28(18):i333-9. https://doi.org/10.1093/bioinformatics/bts378.

115. Anderson CA, Pettersson FH, Clarke GM, Cardon LR, Morris AP, Zondervan KT. Data quality control in genetic casecontrol association studies. Nat Protoc. 2010;5(9):1564-73. Available from: https://pubmed.ncbi.nlm.nih.gov/21085122/. https://doi.org/10.1038/nprot.2010.116.

116. Purcell S, Neale B, Todd-Brown K, Thomas L, Ferreira MAR, Bender D, et al. PLINK: A tool set for whole-genome association and population-based linkage analyses. Am J Hum Genet. 2007;81:559-75 Available from: /pmc/articles/ PMC1950838/.

117. Michigan Imputation Server [Internet]. [cited 2021 Sep 7]. Available from: https://imputationserver.sph.umich.edu/index html\#!pages/home

118. Das S, Forer L, Schönherr S, Sidore C, Locke AE, Kwong A, et al. Next-generation genotype imputation service and methods. Nat Genet. 2016:48(10):1284-7. Available from: https://pubmed.ncbi.nlm.nih.gov/27571263/. https://doi.org/1 0.1038/ng.3656.

119. Auton A, Abecasis GR, Altshuler DM, Durbin RM, Bentley DR, Chakravarti A, et al. A global reference for human genetic variation. Nature. 2015;526:68-74 Available from: https://pubmed.ncbi.n/m.nih.gov/26432245/. 
120. Sudmant PH, Rausch T, Gardner EJ, Handsaker RE, Abyzov A, Huddleston J, et al. An integrated map of structural variation in 2504 human genomes. Nature. 2015;526:75-81 Available from: https:/pubmed.ncbi.nlm.nih.gov/26432246/.

121. Loh PR, Danecek P, Palamara PF, Fuchsberger $C$, Reshef YA, Finucane HK, et al. Reference-based phasing using the Haplotype Reference Consortium panel. Nat Genet. 2016;48(11):1443-8. Available from: https://pubmed.ncbi.nlm.nih. gov/27694958/. https://doi.org/10.1038/ng.3679.

122. Kent WJ. BLAT - The BLAST-like alignment tool. Genome Res. 2002;12(4):656-64.

123. Parsons JD. Miropeats: graphical DNA sequence comparisons. Bioinformatics. 1995;11(6):615-9. Available from: https://a cademic.oup.com/bioinformatics/article-lookup/doi/10.1093/bioinformatics/11.6.615.

124. Jurka J. Repeats in genomic DNA: mining and meaning. Curr Opin Struct Biol. 1998;8(3):333-7. https://doi.org/10.1016/ S0959-440X(98)80067-5.

125. RepeatMasker Home Page [Internet]. [cited 2021 Sep 7]. Available from: http://www.repeatmasker.org/.

126. Dobin A, Davis CA, Schlesinger F, Drenkow J, Zaleski C, Jha S, et al. STAR: Ultrafast universal RNA-seq aligner. Bioinformatics. 2013;29(1):15-21. https://doi.org/10.1093/bioinformatics/bts635.

127. Andrews S, Krueger F, Seconds-Pichon A, Biggins F, Wingett S, FastQC. A quality control tool for high throughput sequence data. Babraham Bioinformatics. Babraham Inst. 2015.

128. Liao Y, Smyth GK, Shi W. FeatureCounts: an efficient general purpose program for assigning sequence reads to genomic features. Bioinformatics. 2014;30(7):923-30. https://doi.org/10.1093/bioinformatics/btt656.

129. Love MI, Huber W, Anders S. Moderated estimation of fold change and dispersion for RNA-seq data with DESeq2. Genome Biol. 2014;15(12):1-21. https://doi.org/10.1186/s13059-014-0550-8.

130. Li S. GeneOverlap. Geneoverl. Test Vis. gene overlaps. 2019; Available from: https:/www.bioconductor.org/packages/ release/bioc/html/GeneOverlap.html.

131. Yu G, Wang LG, Han Y, He QY. ClusterProfiler: an R package for comparing biological themes among gene clusters. Omi A J Integr Biol. 2012;16(5):284-7. https://doi.org/10.1089/omi.2011.0118.

132. Edgar R, Domrachev M, Lash AE. Gene Expression Omnibus: NCBI gene expression and hybridization array data repository. Nucleic Acids Res. 2002;30(1):207-10. https://doi.org/10.1093/nar/30.1.207.

133. Geo accession viewer [Internet]. National Center for Biotechnology Information. U.S. National Library of Medicine; [cited 2021 Sep 7]. Available from: https://www.ncbi.nlm.nih.gov/geo/query/acc.cgi?acc=GSE178206.

134. Yihui-Zhu. Yihui-zhu/epigenetics_genetics_asd_biomarker: placental epigenetics and genetics signatures for early biomarker identification and functional analysis in autism spectrum disorder (ASD) using WGBS, WGS, and RNA-seq [Internet]. GitHub. [cited 2021 Sep 7]. Available from: https://github.com/Yihui-Zhu/Epigenetics_Genetics_ASD_Bioma rker.

\section{Publisher's Note}

Springer Nature remains neutral with regard to jurisdictional claims in published maps and institutional affiliations.

\section{Ready to submit your research? Choose BMC and benefit from:}

- fast, convenient online submission

- thorough peer review by experienced researchers in your field

- rapid publication on acceptance

- support for research data, including large and complex data types

- gold Open Access which fosters wider collaboration and increased citations

- maximum visibility for your research: over $100 \mathrm{M}$ website views per year

At $\mathrm{BMC}$, research is always in progress.

Learn more biomedcentral.com/submissions 